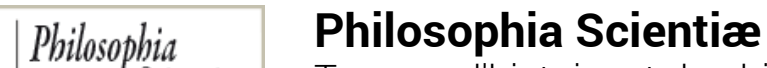

Scientie

Travaux d'histoire et de philosophie des sciences

$14-2$ | 2010

Louis Rougier, De Torricelli à Pascal

\title{
De Torricelli à Pascal
}

Introduction

\section{Simone Mazauric}

\section{(2) OpenEdition}

Journals

Édition électronique

URL : http://journals.openedition.org/philosophiascientiae/172

DOI : 10.4000/philosophiascientiae. 172

ISSN : $1775-4283$

Éditeur

Éditions Kimé

\section{Édition imprimée}

Date de publication : 1 octobre 2010

Pagination : 1-44

ISBN : 978-2-84174-536-4

ISSN : 1281-2463

Référence électronique

Simone Mazauric, «De Torricelli à Pascal », Philosophia Scientiæ [En ligne], 14-2 | 2010, mis en ligne le

01 octobre 2013, consulté le 15 janvier 2021. URL : http://journals.openedition.org/

philosophiascientiae/172 ; DOI : https://doi.org/10.4000/philosophiascientiae.172 


\section{De Torricelli à Pascal Introduction}

Simone Mazauric

Laboratoire d'Histoire des Sciences et de Philosophie, Archives H. Poincaré (UMR 7117), Nancy-Université

\section{1 - Introduction}

Le manuscrit De Torricelli à Pascal est un manuscrit que, pour des raisons que nous ignorons, Louis Rougier a renoncé ou a dû renoncer à publier, faute peut-être d'éditeur. Retrouvé par Denis Lelarge ${ }^{1}$ dans le fonds Rougier conservé à Lourmarin ${ }^{2}$, la revue Philosophia Scientice a choisi d'en assurer la publication, trente ans ou presque après la mort de son auteur. Pourquoi ce choix? Quel intérêt présente ce manuscrit?

Cet intérêt est triple. À commencer par le plus manifeste, et parce que Rougier s'y fait historien des sciences, ce manuscrit, sans doute rédigé en 1927, permet de découvrir un aspect presque totalement ignoré de l'œuvre de Rougier ${ }^{3}$. Hormis en effet un article publié en 1931 [Rougier 1931b, 513-553], et qui traite au moins apparemment de la même question que le manuscrit, rien ne permet de pressentir un quelconque intérêt de Rougier pour une discipline qu'il n'a ainsi, si l'on ne considère que son œuvre publiée, pratiquée que très occasionnellement. Or De Torricelli à Pascal relève doublement de l'histoire des sciences. Rougier y relate

Philosophia Scientice, 14 (2), 2010, 1-45.

1. Laboratoire d'Histoire des Sciences et de Philosophie, Archives H. Poincaré (UMR 7117), Nancy-Université.

2. C. Berndt \& M. Marion signalent l'existence de ce manuscrit dans «Vie et œuvre d'un rationaliste engagé : Louis Rougier (1889-1982)», [Pont \& Padovani 2006].

3. Pour une présentation à la fois synthétique et détaillée de l'œuvre de Rougier, voir Claudia Berndt et Mathieu Marion, art. cit., et plus généralement [Pont \& Padovani 2006, 2007]. Nous ne reviendrons pas dans cette introduction sur de nombreux aspects de la vie et de l'œuvre de Rougier, qui sont traités dans ces deux volumes, auxquels nous nous contentons de renvoyer le lecteur. 
en effet la façon dont les travaux de Torricelli puis de Pascal ont permis, dans les années 1646-1648, d'établir, contre le dogme aristotéloscolastique de l'horror vacui, l'existence du vide, et de mettre en évidence le rôle joué par la pression atmosphérique pour rendre compte des phénomènes jusque-là imputés à cette prétendue horreur du vide. Ce manuscrit constitue donc une contribution originale et inédite au traitement d'une question à laquelle les historiens des sciences, depuis le XVII ${ }^{\mathrm{e}}$ siècle, n'ont jamais cessé de s'intéresser ${ }^{4}$. Toutefois, la « querelle du vide » n'a jamais été simplement une querelle d'ordre théorique. Sur la querelle proprement scientifique, se sont en effet très vite greffées plusieurs querelles de priorité, et notamment celle déclenchée par le biographe de Descartes, Adrien Baillet, qui, dès 1691, affirmait que c'était Descartes, et non pas Pascal, qui avait le premier conçu l'idée de l'expérience dite du puy de Dôme ${ }^{5}$. Cette querelle de priorité a été relancée de façon extrêmement polémique au début du $\mathrm{XX}^{\mathrm{e}}$ siècle, quand Félix Mathieu a publié en 1906 dans la Revue de Paris un article dans lequel il accusait Pascal de s'être indûment approprié non seulement l'idée de cette expérience, mais aussi celle de l'expérience dite du vide dans le vide, due selon lui à Adrien Auzoult ${ }^{6}$. En riposte à cette accusation lancée contre Pascal, de nombreux historiens des sciences sont alors revenus à nouveaux frais sur un épisode dont la complexité autorise il est vrai diverses interprétations ${ }^{7}$. Surtout, depuis 1906, il est devenu presque impossible de parler des travaux de Torricelli et de Pascal sans prendre parti dans cette «méta-querelle» et sans statuer sur la sincérité ou l'insincérité de $\mathrm{Pascal}^{8}$ : c'est ce que ne manque pas de faire à son tour

4. On trouve une bibliographie très complète sur le sujet dans [Mesnard 1970, II, 673-676]. Depuis 1970, d'autres ouvrages sont parus sur cette même question, notamment [Mazauric 1998].

5. Sur cette querelle aux ramifications multiples, voir [Mazauric 1998].

6. [Mathieu 1906]. Il s'agit en fait d'un seul article, publié en trois livraisons successives.

7. Voir à ce sujet la bibliographie proposée par Mesnard [Mesnard 1970].

8. C'est encore le cas aujourd'hui où l'on ne peut prétendre qu'un consensus soit établi en ce qui concerne les faits controversés. Cependant, la possibilité d'une retouche par Pascal de la lettre envoyée à Florin Périer est assez largement admise. En revanche, Sylvain Matton va plus loin que Félix Mathieu et pense que Pascal a également retouché la relation de l'expérience du puy de Dôme envoyée par Périer [Matton 1994, 2004]. Sans entrer dans le détail de la controverse, il paraît évident que la lettre de Pascal est adressée au public, et a été rédigée dans cette intention; et que bien des questions posées par Félix Mathieu demeurent pertinentes. L'hypothèse de Sylvain Matton de son côté est très crédible. Tout le monde en revanche s'accorde à reconnaître les inexactitudes factuelles contenues dans la Lettre à $M$. de Ribeyre, lettre dans laquelle Pascal se disculpait de l'accusation de s'être indûment approprié l'expérience de Torricelli (voir plus loin). En outre, depuis la rédaction du manuscrit de Rougier, d'autres accusations ont été adressées à Pascal, par Alexandre Koyré no- 
Rougier, aussi bien dans le manuscrit que dans l'article du Mercure de France. La publication de ce manuscrit permet par conséquent à la fois d'ajouter à la connaissance que les spécialistes de Louis Rougier peuvent avoir de son œuvre et de découvrir la façon, singulière à certains égards, ainsi que nous le montrerons plus loin, dont Rougier a fait œuvre, fût-ce épisodiquement, d'historien des sciences.

Toutefois, peut-on véritablement considérer que l'article du Mercure de France et le manuscrit relèvent tous les deux et exclusivement de l'histoire des sciences? Le titre de l'article «L'affaire Pascal et la méthode littéraire de M. Brunschvicg » pose en effet la question de savoir quel est le but véritable ou du moins le but principal qu'a poursuivi Rougier en le publiant : traiter simplement, sur un mode beaucoup plus condensé que le manuscrit, un épisode célèbre de l'histoire des sciences ou polémiquer contre Léon Brunschvicg? À le lire un peu rapidement, l'article du Mercure de France peut passer en effet pour une sorte de résumé de De Torricelli à Pascal, dont il reprend d'ailleurs quelques formulations : une façon de réemployer les matériaux d'une recherche qui n'a pas finalement débouché sur une publication? Nous y reviendrons. Le manuscrit lui-même soulève une question semblable mais un peu différente. L'Avertissement et la Conclusion le laissent clairement entendre, c'est moins sans doute la question du vide à proprement parler ou celle de la sincérité/insincérité de Pascal qui intéressent Rougier que la façon dont la question du vide et de la pression atmosphérique a été traitée au XVII ${ }^{\mathrm{e}}$ siècle, car cette façon illustre selon lui clairement la défaite de la science scolastique. Or, on le sait, Rougier a été un ardent artisan du combat contre le renouveau du thomisme, impulsé dans toute l'Europe catholique par la promulgation, en 1879, par le pape Léon XIII, de l'Encyclique Aeterni Patris ${ }^{9}$, et relayé en France par des intellectuels et philosophes comme Jacques Maritain ou Étienne Gilson. En 1925, Rougier publiait La Scolastique et le Thomisme [Rougier 1925a], ouvrage dans lequel il s'employait très longuement à exposer les raisons de la «faillite philosophique » que représentait à ses yeux la scolastique ${ }^{10}$. On peut dès lors se demander si traiter de la question du vide et de la pression atmosphérique telle qu'elle a été posée au XVII ${ }^{\mathrm{e}}$ siècle n'était pas pour

tamment, qui a fortement mis en question la réalisation de certaines des expériences de Rouen [Koyré 1956]. Enfin, plus récemment, Kimiyo Koyanagi [Koyanagi 1989] a repris cette accusation en la radicalisant. Autant dire que ce thème de recherche n'a pas fini d'être exploré.

9. Aeterni Patris. De philosophia christiana ad mentem S. Thomae Aquinatis, Doctoris Angelici, in scholis catholicis instauranda.

10. L'ouvrage a été réédité sous un format réduit en 1966 sous un titre beaucoup plus explicite : Histoire d'une faillite philosophique : la Scolastique, [Rougier 1925b]. 
Rougier moins une fin en soi qu'un moyen de poursuivre l'offensive entamée avec La Scolastique et le Thomisme, dont le manuscrit aujourd'hui publié offre ainsi un prolongement. Du même coup d'ailleurs, ce manuscrit se dépouille de son caractère singulier et isolé pour s'insérer très logiquement dans l'œuvre globale de Rougier, qu'il permet de cette façon également de mieux connaître.

En même temps et enfin, la très vive controverse déclenchée autour de Pascal et de ses travaux sur le vide et la pression atmosphérique par Félix Mathieu ne prend sens que dans la conjoncture à la fois politique, philosophique et idéologique du tournant des $\mathrm{XIX}^{\mathrm{e}}$ et $\mathrm{XX}^{\mathrm{e}}$ siècles, très marquée par l'affaire Dreyfus, et par l'affrontement entre républicains laïcs et spiritualistes plus ou moins conservateurs, sur fond de restauration du thomisme et de querelle autour de la modernité, une querelle encore une! - dont la position de ses protagonistes à l'égard de Pascal constituait un marqueur particulièrement significatif ${ }^{11}$. Sous cet aspect également, et dans la mesure où l'un des représentants majeurs du spiritualisme universitaire, Léon Brunschvicg, a joué un rôle particulièrement important dans cette conjoncture en procédant à partir de 1908, en collaboration avec Émile Boutroux, à l'édition des œuvres de Pascal, et en raison des rapports particuliers noués par Rougier avec le philosophe, la découverte de ce manuscrit, accompagnée de la lecture de l'article du Mercure de France, apporte un éclairage intéressant sur une conjoncture singulière sur laquelle il contraint à revenir et à mieux percevoir dans toute sa complexité, en même temps que cette conjoncture projette réciproquement un éclairage particulier sur De Torricelli à Pascal.

Texte très polysémique, dont les significations ne cessent de s'entrecroiser, De Torricelli à Pascal intéresse ainsi aussi bien les historiens des sciences que les spécialistes de l'œuvre de Rougier, et tout autant les historiens des idées mais aussi les historiens «tout court» des premières décennies du $\mathrm{xx}^{\mathrm{e}}$ siècle. Ce manuscrit méritait par conséquent amplement d'être enfin édité.

\section{2 - L'histoire des sciences dans l'œuvre de Rougier}

Le manuscrit intitulé De Torricelli à Pascal se donne d'abord et avant tout comme un ouvrage d'histoire des sciences, plus précisément comme un ouvrage d'histoire de la physique à l'époque moderne, où il est question du vide et de la pression atmosphérique, plus généralement de

11. À ce sujet, voir [Compagnon 2007]. 
«l'équilibre des liqueurs », c'est-à-dire de ce que l'on nommera plus tard l'hydrostatique. Ces deux questions ont été en effet historiquement posées ensemble dans les travaux de Torricelli puis de Pascal, même si elles sont sur le plan théorique parfaitement indépendantes l'une de l'autre. En même temps, il y est question de la priorité contestée de Pascal. Un tel constat pose d'emblée et doublement la question de la place de ce manuscrit dans l'œuvre de Rougier.

\section{1 - La datation de De Torricelli à Pascal}

Cette question peut s'entendre d'abord en un sens chronologique. Aucune indication explicite ne permet de le dater avec certitude. Il est cependant possible de déterminer avec une très grande probabilité le moment où il a été rédigé, grâce d'abord aux indications que fournit l'article du Mercure de France publié en 1931 sur le même sujet. L'article est paru alors que le manuscrit, lui, n'est pas paru, Louis Rougier s'en explique au moins partiellement dans une longue note de l'article [Rougier 1931b, 544, n. 38], où il délivre quelques indications concernant la place respective du manuscrit et de l'article. Il raconte en effet qu'il préparait un ouvrage sur «La Déroute de la scolastique au XVII ${ }^{\mathrm{e}}$ siècle — - c'est le texte qui deviendra De Torricelli à Pascal —, mais qu'il a renoncé à publier ce manuscrit, qui est venu prendre place dans ses papiers posthumes. À la place de la publication projetée, il a choisi de faire paraître, quelques années plus tard, l'article du Mercure de France, venu ainsi se substituer, au moins partiellement, dans la mesure où il est nettement plus bref, à la parution du manuscrit. De cette note, on doit déduire que De Torricelli à Pascal a été rédigé immédiatement après La Scolastique et le Thomisme, dont il devait être le prolongement, sans doute par conséquent vers 1926 ou 1927, et que la rédaction de celui-ci a précédé celle de l'article, pour la rédaction duquel Rougier a réemployé une partie de son manuscrit. Nous constaterons ultérieurement d'autres réemplois de ce manuscrit. C'est ce que confirme l'Avertissement de ce dernier, qui contient quelques indications concordantes sur la date probable de sa rédaction ainsi que sur les raisons pour lesquelles Rougier vient de se décider à le publier :

... il nous convient de dire pourquoi, ayant renoncé à la publication de ce volume, terminé il y a quatre ans, nous nous décidons aujourd'hui à lui laisser voir le jour ${ }^{12}$.

12. [Rougier 2010, 49]. Il est évident que cette publication annoncée n'a finalement pas eu lieu. 
Ce dont on peut déduire que Rougier a bien eu à un certain moment l'intention ferme de publier ce manuscrit et si celui-ci a été achevé vers 1926 ou 1927 au plus tard, il aurait eu l'intention de le publier vers 1931, après l'avoir gardé par devers lui durant ces quatre ans. Or 1931 est la date de publication de l'article du Mercure de France, ce qui confirme que c'est faute d'avoir pu effectivement publier son manuscrit qu'il a publié l'article «L'affaire Pascal et la méthode littéraire de M. Brunschvicg ». Mais on peut supposer qu'une circonstance supplémentaire a pu susciter cette publication. On relèvera d'ailleurs que Rougier a non seulement réutilisé le manuscrit de De Torricelli à Pascal en en tirant un article, mais il a un peu auparavant, dans un article publié également dans le Mercure de France [Rougier 1931a], réemployé une petite partie de ce même manuscrit. Nous le signalerons ci-après.

\section{2 - De Torricelli à Pascal et «L'affaire Pascal et la méthode littéraire de M. Brunschvicg »}

La question chronologique étant réglée, est posée celle de la place occupée par un ouvrage d'histoire des sciences dans l'œuvre d'un philosophe qui n'a pas, et à juste titre, la réputation d'un historien des sciences. Ses travaux publiés témoignent certes d'un réel éclectisme puisqu'ils concernent aussi bien la philosophie des sciences que la philosophie politique ou l'histoire des religions ${ }^{13}$. Et en tant que philosophe des sciences, Rougier s'est intéressé à la physique. Mais uniquement à la physique la plus récente : relativité, physique quantique. Le manuscrit aujourd'hui publié peut donc sembler n'entretenir de rapport avec l'œuvre dans son ensemble que marginalement. Seul l'article du Mercure de France dont il vient d'être question paraît relever de l'histoire des sciences. Paraît, car cette catégorisation est en partie problématique. On peut en effet légitimement se demander quel est le statut exact de cet article qui semble répondre au moins autant à une intention polémique dirigée contre Léon Brunschvicg qu'à une intention historienne : le titre même de l'article témoigne de cette dualité d'intention. Quant à De Torricelli à Pascal, la question de son appartenance pleine et entière à l'histoire des sciences se pose également, même si c'est à un moindre degré et d'une tout autre façon. Quel est donc d'abord le statut exact de l'article du Mercure de

13. Selon Jean-Claude Pont [Pont \& Padovani 2007, 11], Rougier distinguait luimême dans son œuvre sept sections : Théorie de la connaissance, Logique, Philosophie scientifique, Histoire de la philosophie et des Religions, Science politique, Économie politique, Histoire contemporaine. On constate sans peine que l'histoire des sciences ne figure pas parmi ces rubriques. 
France? Et quel est le rapport qu'entretiennent les deux textes? L'article du Mercure de France est-il un simple résumé du manuscrit ou bien les deux textes se différencient-ils non seulement par leurs dimensions mais aussi par des décalages théoriques suffisants pour qu'ils empêchent de se rallier à la première hypothèse? Répondre à la seconde question aidera également à répondre à la première.

\subsection{1 - De Torricelli à Pascal : le débat scientifique et « l'affaire Pascal »}

En ce qui concerne les rapports entretenus par le manuscrit et l'article du Mercure de France, et même si Rougier paraît avoir publié le second en lieu et place du premier, il est certain que celui-là n'est ni le simple résumé ni le succédané de celui-ci.

Pour établir ce point, il faut détailler davantage le contenu des deux textes et reprendre l'exposé de la double querelle qu'ils ont tous les deux pour objet. De Torricelli à Pascal entremêle en effet, ce qui était devenu presque inévitable depuis la publication des articles de Félix Mathieu, deux questions différentes. D'une part, il se présente comme le récit des travaux de Torricelli, puis surtout de Pascal, pour établir l'existence $\mathrm{du}$ vide et pour montrer comment les phénomènes jusqu'alors imputés à l'horreur du vide (comme par exemple la montée de l'eau dans les pompes vides, phénomène qui, observé dans ses limites par les fontainiers de Florence, a été à l'origine des travaux de Torricelli puis de Pascal) s'expliquent non pas par l'horror vacui, mais par la pression exercée par l'air environnant sur la surface de l'eau que l'on veut faire monter dans les pompes. Cette seconde hypothèse, celle du poids et de la pression de l'air, comme cause à la fois de la montée de l'eau dans les pompes vides mais aussi des limites de cette montée, imputables à la limite du poids et de la pression de l'air, a d'abord été émise par Torricelli, et mise en évidence à l'aide du dispositif expérimental qu'il a imaginé ${ }^{14}$. Elle a été vérifiée expérimentalement en 1648 d'abord par l'expérience dite du vide dans le vide, puis par l'expérience effectuée par Florin Périer, le beau-frère de Pascal, et à la demande de ce dernier, au sommet du puy de Dôme. Pascal, dans la lettre qu'il dit avoir écrite le 15 novembre 1647 à son beau-frère, Florin Périer, pour lui demander d'effectuer cette expérience, se présente comme celui non seulement qui a établi le premier l'existence

14. Dans un tube d'un mètre rempli de mercure, renversé sur une cuve elle-même remplie de mercure, le mercure descend puis s'immobilise à la hauteur de 76 centimètres. C'est cette expérience qui est habituellement désignée sous le nom d'« expérience ordinaire du vide ». 
du vide, mais aussi comme celui qui a eu le premier l'idée de ces deux expériences qu'il a lui-même réalisée, pour la première, ou fait réaliser par son beau frère, pour la seconde ${ }^{15}$.

Il est difficile de raconter cette histoire, on le constate, sans attirer l'attention, voire sans prendre parti sur les différentes questions de priorité qu'un examen attentif de cet épisode soulève nécessairement. Car les premiers travaux et les premières expériences sur le vide, ainsi que l'hypothèse de la pression atmosphérique sont en Italie, dans les premières années de la décennie 1640 , le fait des élèves et amis de Galilée ${ }^{16}$, puis surtout de Torricelli. Et en France, à partir de 1646, d'autres savants que Pascal s'intéressent à la question du vide. C'est le cas du père Mersenne, qui a eu le premier connaissance des expériences réalisées en Italie et les a fait connaître en France. C'est le cas de Roberval, d'Auzoult, un ami de Pascal, de Descartes, qui certes vit en Hollande, mais qui manifeste un grand intérêt pour cette question et qui, partisan du plein mais favorable à l'hypothèse de la «pesanteur et pression de l'air », a rencontré Pascal à Paris à l'occasion du voyage qu'il a accompli en septembre 1647, et a toujours affirmé avoir, lors de ces rencontres, suggéré à Pascal l'idée d'accomplir l'expérience dite ordinaire du vide à des altitudes différentes, afin de vérifier si le mercure diminue bien dans le tube barométrique lorsqu'on s'élève en altitude en raison de la diminution de la «pesanteur et pression de l'air » exercée sur le mercure contenu dans la cuvette où le tube est plongé. Un jésuite, le père Daniel, a le premier reconnu la légitimité des revendications de Descartes, affirmant que l'expérience du puy de Dôme devrait bien plutôt s'appeler «l'expérience de Descartes ${ }^{17}$ » et Adrien Baillet, à son tour, a proclamé les droits de ce dernier :

L'expérience du puy de Dôme fut faite sur les avis de monsieur Descartes quoique monsieur Pascal l'ait dissimulé. [Baillet 1691]

Quant à l'expérience dite «du vide dans le vide», elle a été réalisée sous trois formes différentes : celle que décrit Pascal, mais Auzoult et

15. Il est impossible, dans le cadre de cette introduction, de prétendre résumer de façon fidèle tous les éléments du débat et toutes les péripéties qui ont ponctué l'établissement des deux propositions centrales. Ni d'évoquer tous les protagonistes de l'affaire. Nous nous contentons d'indications succinctes, forcément incomplètes.

16. Le rôle des élèves de Galilée n'est pas alors très bien connu quand Rougier rédige De Torricelli à Pascal, c'est pourquoi il n'en parle pas.

17. [Daniel 1691]. Le père Daniel est évidemment animé d'une intention antijanséniste et ne se contente pas de revendiquer la priorité de Descartes contre Pascal, auquel il reproche également d'avoir largement emprunté, dans son Traité des coniques, au géomètre Desargues. 
Roberval l'ont réalisée également, à l'aide de dispositifs expérimentaux chaque fois différents, [Mouy 1934]. Et à peu près au même moment, sans que l'on puisse assigner avec certitude à chacune de ces expériences une date précise.

Ces expériences, celles de Pascal, de Mersenne, de Roberval, d'Auzoult, les prises de position théoriques des uns et des autres, la question même de savoir si Descartes avait bien suggéré l'idée de l'expérience à Pascal, ont fait l'objet, depuis le XVII ${ }^{\mathrm{e}}$ siècle, de plusieurs récits. Ceux d'abord du père Daniel et de Baillet, puis celui de Montucla [Montucla 1758] et, pour se rapprocher du Xx ${ }^{\mathrm{e}}$ siècle, celui de Charles Thurot [Thurot 1872], de J.-F. Nourrisson [Nourrisson 1881], celui de Ernest Havet [Havet, E. 1885], de Joseph Bertrand [Bertrand 1891], de Charles Adam [Adam 1887, 1888]. L'article publié en trois parties en 1906 dans la Revue de Paris par Félix Mathieu prend ainsi place dans une longue tradition historiographique, et il est loin de ne poser que des questions inédites. En revanche, il opère une rupture très sensible avec la tradition historiographique antérieure dans la mesure où, à la différence de ses prédécesseurs, Félix Mathieu adopte un ton inhabituellement polémique, pour des articles d'histoire des sciences s'entend. Car si son long article est un article très savant, très érudit, reposant sur une documentation très riche et témoignant d'une connaissance solide des faits relatés, son ton est délibérément accusateur et il constitue un long, persévérant et systématique réquisitoire dirigé contre Pascal. Tout du long, les jugements dépréciatifs abondent : Pascal est estimé 《hautain », «impitoyable», 《 impoli », parfois même « injurieux », « cruel» en tout cas, en outre 《 orgueilleux », « vaniteux », «intolérant ». Pire encore, si l'on considère le débat de fond, dénué de toute originalité sur le plan scientifique : on ne trouve pas en effet selon Félix Mathieu « la moindre idée neuve et utile » dans les premières expériences effectuées par Pascal à Rouen, et ces expériences par conséquent «n'ajoutent rien à ce qu'avait dit Galilée » [Mathieu 1906]. Mais de ce sévère réquisitoire, se détache l'accusation principale, résumée et énoncée avec vigueur dans une conclusion devenue immédiatement célèbre :

La lettre que Pascal dit avoir écrite, le 15 septembre 1647, à son beau-frère Périer, pour le prier de monter sur le Puy-deDôme est un faux, et ce faux est le couronnement de tout un système d'artifices par lequel Pascal a tenté de s'approprier l'hypothèse de la pression atmosphérique, que nous devons à Képler, Isaac Beeckman, Baliano et Torricelli, et a réussi à s'approprier les inventions qui apportèrent la vérification 
expérimentale de cette hypothèse : l'expérience du vide dans le vide, qui appartient à Auzoult, et l'idée de l'expérience du Puy-de-Dôme, qui appartient à Descartes. [Mathieu 1906, $1^{\text {er }}$ mai, 206]

C'est évidemment cette double accusation qui déclenche un tollé et suscite durant deux ans à un rythme soutenu la publication de très nombreux articles parus dans les revues les plus célèbres en France et parfois à l'étranger, dans lesquels partisans et adversaires de Félix Mathieu s'affrontent ${ }^{18}$. Les adversaires sont beaucoup plus nombreux que les partisans, et parmi ces derniers bien peu, pour ne pas dire aucun, ne soutient réellement ou en totalité la thèse défendue par Félix Mathieu : certains cependant concèdent ou reconnaissent que ce dernier a bien soulevé quelques questions embarrassantes ${ }^{19}$. Parmi ceux qui prennent parti dans la querelle, l'un des premiers à intervenir est Léon Brunschvicg, qui préparait alors, en collaboration avec Émile Boutroux, l'édition des Euvres de Pascal ${ }^{20}$, [Brunschvicg \& Boutroux 1908-1914], [Brunschvicg $1906,1907]$ et qui, tout en rendant hommage aux recherches savantes et minutieuses de Félix Mathieu, en récuse totalement les conclusions tout en lui reprochant de n'avoir pas respecté les règles de la méthode historique. Passés deux ans d'intense polémique, celle-ci s'apaise et les publications sur la question se raréfient considérablement.

Quand il se penche à son tour sur la question du vide et de la pression atmosphérique à la fin des années 1920, Rougier ne peut pas ignorer cette mise en accusation de Pascal par Félix Mathieu, il ne peut pas ignorer l'acuité de la querelle de priorité que celui-ci a relancée, — pas plus qu'aucun des historiens des sciences qui se penchent depuis sur cette question -, il ne peut pas davantage ignorer l'existence de cette très importante littérature, à laquelle il fait référence à de nombreuses reprises, et notamment dans l'Avertissement et dans la Conclusion, mais

18. Voir à ce sujet la bibliographie établie par Jean Mesnard, [Mesnard 1970]. Il faut ajouter pour bien comprendre la réalité même de cette querelle qu'il n'existe aucun autographe de cette lettre, que Pascal a publiée dans le Récit de la grande expérience de l'équilibre des liqueurs, Paris, 1648. C'est lui par conséquent qui donne pour cette lettre la date de novembre 1647, qu'il est impossible de vérifier.

19. Celle notamment qui concerne la façon dont Pascal a reconnu, sur un mode pour le moins singulier, sa dette à l'égard de Torricelli. À ce sujet, voir [Mazauric 2009]. D'une façon plus générale, il serait intéressant de se livrer à une analyse détaillée des différents articles parus à la suite de la publication des articles de Félix Mathieu, et de distinguer les positions de tous ceux qui sont intervenus dans la querelle, mais une telle analyse outrepasserait largement les bornes de cette introduction. À ce sujet, voir cependant n. 28 infra.

20. Le tome I et le tome II paraîtront en 1908. 
dont il retient surtout la défense de Pascal par Léon Brunschvicg et la querelle spécifique qui a opposé ce dernier à Félix Mathieu. Rougier intervient donc sur cette double question ${ }^{21}$.

De Torricelli à Pascal traite ainsi simultanément une question d'histoire des sciences « à proprement parler», celle de la façon dont la question du vide et de la pression atmosphérique a été traitée au XVII ${ }^{\mathrm{e}}$ siècle, et celle du bien-fondé de la mise en accusation de Pascal. En ce qui concerne cette seconde question, Rougier dit tenir à marquer son indépendance à l'égard des positions de Félix Mathieu, qu'il ne partage que partiellement. En d'autres termes, si Rougier traite bien les deux questions, il ne laisse pas cependant la seconde prendre le pas sur la première, qu'il traite de façon approfondie, et maintient entre les deux un réel équilibre. Tout au plus peut-on constater que Brunschvicg est déjà, parmi les partisans de Pascal, celui contre lequel il polémique principalement.

\subsection{2 - «L'affaire Pascal et la méthode littéraire de M. Brunschvicg »}

Ce qui n'est pas du tout le cas dans l'article du Mercure de France, où la question proprement historique n'occupe plus la première place et dans lequel Rougier prend surtout pour cible le rôle joué par Léon Brunschvicg dans «l'affaire Pascal». C'est ce que disent explicitement à la fois le titre et les premières lignes de l'article :

L'affaire Pascal nous présente aujourd'hui l'occasion d'examiner la méthode littéraire de M. Brunschvicg.

Autant dire que «l'affaire Pascal» n'est plus dans cet article qu'un prétexte pour polémiquer d'abord contre l'université française en général, accusée d'avoir pris massivement le parti de Pascal, et plus encore contre Brunschvicg en particulier et principalement. Contre l'université française dénoncée comme la gardienne de l'orthodoxie, de la tradition : Rougier utilise sciemment un vocabulaire clérical $^{22}$ pour décrire le rôle joué par les partisans de Pascal dans l'《affaire» :

On n'eut de cesse qu'on n'eût réuni une sorte de Saint-Office universitaire. Le tribunal se composa de MM. Lefranc ${ }^{23}$,

21. On peut penser que choisir comme objet d'étude une double querelle, de la part d'un homme dont la vie a été ponctuée de polémiques, ne relève peut-être pas du hasard.

22. À ce sujet, voir plus loin.

23. [Lefranc 1906]. Il est intervenu encore trois fois sur cette question. 
Louis Havet ${ }^{24}$, Gabriel Monod ${ }^{25}$, Gaston Milhaud ${ }^{26}$, Duhem $^{27}$, Strowski ${ }^{28}$, Brunschvicg. Contre l'hérétique, on oublia les petites nuances doctrinales pour fulminer, d'un commun accord, une excommunication majeure. M. Mathieu fut convaincu d'opinions téméraires et erronées, et d'avoir malignement, méchamment et impudemment suspecté, altéré, faussé et outragé la vérité. [Rougier $1931 b^{29}$ ]

Mais une affaire est un mot qui relève aussi du champ politique, et qui a amplement servi au moment où Félix Mathieu publiait ses articles. L'affaire Pascal fait inévitablement penser à l'affaire Dreyfus, et pour cause, comme le précise Rougier dans les Conclusions de son manuscrit : « il est impossible d'en entreprendre l'historique ${ }^{30}$ sans rencontrer l' « affaire Pascal », de laquelle M. Brunschvicg n'hésitait pas à rapprocher, dans le second bulletin de l'Union pour la vérité, l' «affaire Dreyfus ${ }^{31} »$. Il rappelle à nouveau dans l'article que « M. Brunschvicg trouva à pro-

24. [Havet, L. 1907].

25. [Monod, 1907].

26. [Milhaud 1907]. Il est étonnant de constater que Gaston Milhaud est revenu quelques années plus tard [Milhaud 1918] sur la question de la priorité respective de Descartes et de Pascal dans l'invention de l'expérience qui deviendra l'expérience du puy de Dôme, et qu'il ne fait alors aucune allusion à l'article de la Revue scientifique, tout en affirmant clairement que Descartes a bien conçu l'idée de cette expérience et l'a suggérée à Pascal. Même s'il est un peu rapide de parler de contradiction entre deux textes qui n'ont pas le même objet et ne poursuivent pas les mêmes objectifs, on ne peut que s'étonner au moins du silence de Milhaud sur la querelle dont il a été un protagoniste actif.

27. [Duhem 1906a].

28. [Strowski 1907]. L'énumération de ces différents noms est à certains égards trompeuse, car elle donne l'impression que tous ceux que cite Rougier ont unanimement et radicalement condamné les thèses de Félix Mathieu. Les choses ont été en fait un peu plus compliquées. D'un auteur à l'autre, les positions diffèrent autant que le ton de chacun. Il est vrai cependant que tous ceux que cite ici Rougier ont récusé les accusations de Félix Mathieu. Ce qui laisse ignorer que d'autres, il est vrai très peu nombreux, comme le père Thirion et surtout Abel Rey, qui est incontestablement le plus favorable à Félix Mathieu, ont entendu certains des arguments de ce dernier (voir à ce sujet [Rey 1906a, 1906b]), au point qu'il a rendu au passage hommage à ses travaux sur Pascal un peu plus tard et à une tout autre occasion [Rey 1907]. On doit également rappeler ici que les positions de Gaston Milhaud ont apparemment évolué. Encore une fois, une analyse précise des positions des uns et des autres mériterait d'être entreprise.

29. Félix Mathieu est ici à l'évidence implicitement comparé à Galilée, victime de l'intolérance de l'Église et des théologiens, que Rougier dénonce également à l'occasion dans De Torricelli à Pascal.

30. [Du conflit de la physique scolastique avec la science expérimentale].

31. Dans cet article, après avoir indiqué qu'il souhaitait tenir dans l'affaire le rôle du « spectateur impartial et désintéressé qui essaie de mesurer pour son propre compte la valeur des charges qu'on fait peser contre l'accusé », Brunschvicg ajoutait : 
pos d'évoquer l'affaire Dreyfus à l'occasion de l'affaire Pascal » [Rougier 1931b, 517], et ironise sur ce «suggestif parallèle ». Tout en s'emparant de la comparaison, et en la retournant contre l'accusateur, donnant à Brunschvicg le mauvais rôle dans l'« affaire Pascal ». Rougier n'oublie pas pour autant entièrement le contenu scientifique de cette dernière et lui consacre plusieurs parties de l'article, mais pour conclure à la priorité d'Auzoult dans l'exécution de l'expérience du vide dans le vide : c'était la thèse défendue par Félix Mathieu. Et c'est pour mieux revenir in fine à « la méthode littéraire de M. Brunschvicg », à ce qu'il appelle son exégèse, sa «dialectique », une dialectique qu'il n'hésite pas à qualifier de «talmudique ».

On sait, mais nous n'entrerons pas dans l'examen de cette question, qui ne peut être aisément résolue, que l'accusation d'antisémitisme a été lancée à l'encontre de Rougier. Ce qualificatif et l'article dans son ensemble constituent évidemment une pièce à verser au dossier de cet antisémitisme, réel ou supposé ${ }^{32}$. Ce que l'on peut affirmer de façon certaine, c'est que l'article est surtout une charge, très violente, contre Brunschvicg, et Rougier donne d'ailleurs à deviner les raisons personnelles qu'il pouvait avoir de prendre ainsi à parti ce dernier : son absence de position stable dans l'Université française, dont il tenait Brunschvicg

C'est ainsi qu'au lendemain de la publication de l'acte d'accusation contre Dreyfus, le 7 janvier 1898, nous n'étions pas définitivement éclairés sur le fond de l'affaire, mais nous savions ce que valait le procès de 1894 ; nous ne connaissions pas Dreyfus encore moins Esterhazy et ses complices; nous ne connaissions pas M. Besson d'Ormescheville. Nous n'aurons pas autre chose à examiner que les éléments de conviction réunis par M. Mathieu contre Pascal. [Brunschvicg 1906, 144].

32. On peut peut-être verser à ce même dossier l'allusion de Rougier, dans les premières pages de l'article, « aux savantes et lucratives éditions des Opuscules et des Pensées »[Rougier 1931b, 516]. Claudia Berndt et Mathieu Marion traitent de façon assez détaillée, mais un peu hésitante, la question de l'antisémitisme de Rougier. Question difficile, qui oblige à prendre en compte les positions politiques de Louis Rougier, ce qu'il est impossible de faire dans le cadre de cette introduction. Sur le fond, cette question oblige sans doute à distinguer des formes et des degrés dans l'antisémitisme, des dates ou des périodes aussi : avant, pendant ou après le nazisme et les formes extrêmes, c'est-à-dire criminelles et génocidaires, d'antisémitisme qu'il a suscitées. Peut-être serait-il aussi plus pertinent de parler à son propos d'anti-judaïsme, quand on sait la place qu'a tenue dans son œuvre la critique des religions. Disons simplement rapidement que l'antisémitisme de Rougier, si on doit le reconnaître, relevait sans doute de la catégorie de l'antisémitisme « ordinaire» (un qualificatif qui ne le rend pas pour autant acceptable) et s'est sans doute nourri de rancœurs personnelles à l'égard de Brunschvicg. Voir la note suivante. 
pour responsable ${ }^{33}$ et qui autorisait selon lui toutes les tentatives d'intimidation dont il aurait été victime de sa part ${ }^{34}$.

Bref, il n'est finalement qu'à demi question d'histoire des sciences dans cet article, où Rougier règle prioritairement ses comptes avec Brunschvicg, et l'on peut regretter que ce soit finalement l'article que Rougier ait choisi de publier plutôt que le manuscrit où, à l'inverse, l'histoire des sciences, l'histoire de la physique tient la place principale et la polémique une place relativement subordonnée. Et si Brunschvicg est bien également celui contre lequel Rougier dirige ses attaques, cellesci se répartissent cependant assez équitablement entre plusieurs cibles et ne revêtent jamais la forme d'attaques ad hominem.

\section{3 - De Torricelli à Pascal et le thomisme}

Pour autant, même si Rougier traite bien dans ce manuscrit la façon dont Pascal, à la suite ou dans la logique des travaux, on dirait aujourd'hui «pionniers », de Torricelli a posé les fondements de l'hydrostatique, doit-on tenir De Torricelli à Pascal comme un ouvrage d'histoire des sciences à part entière? Ou plus exactement, mais c'est finalement la même question, dans la mesure où Rougier n'a qu'à cette occasion pratiqué l'histoire des sciences, ce manuscrit fait-il figure d'exception dans l'œuvre de Rougier? C'est donc maintenant la question de la place, au sens architectonique du terme, occupée par le manuscrit dans l'œuvre de Rougier qui est posée. L'Avertissement et la Conclusion de l'ouvrage n'autorisent qu'une réponse mitigée à la première question tout en permettant de montrer comment celui-ci s'insère très logiquement dans une œuvre qui a pourtant largement négligé l'histoire des sciences.

L'Avertissement présente en effet explicitement De Torricelli à Pascal comme la suite ou le prolongement de La Scolastique et le Thomisme, l'ouvrage que Rougier avait publié en 1925. Il s'agit donc maintenant de

33. Brunschvicg aurait fait échouer sa candidature en 1930 à la succession de Goblot à Lyon. Ce serait cette circonstance qui aurait finalement décidé Rougier à publier l'article du Mercure de France et qui expliquerait que la question du vide y soit en partie négligée au profit de la polémique contre Brunschvicg (voir à ce sujet, l'article de Berndt \& Marion [Pont \& Padovani 2006]). Cet échec de Rougier explique aussi sans doute ses attaques plus générales contre le « Saint-Office universitaire ».

34. «M. Brunschvicg trouva expédient de faire circuler, dans l'Université, le bruit que j'étais venu l'accuser à tort. Le lecteur conviendra qu'il y avait quelque témérité dans cette façon, d'ailleurs classique, de retourner l'accusation. Mais on spéculait sur ma situation, alors instable, que l'on entendait bien maintenir telle, dans l'espoir, me sachant sans fortune, de me fermer la bouche à jamais », [Rougier 1931b, 543, n. 38]. 
mettre en évidence la façon dont les deux ouvrages se relient effectivement entre eux. Rougier a lui-même indiqué la façon dont l'un fait suite à l'autre :

Après avoir étudié dans notre ouvrage, La Scolastique et le Thomisme, comment la pensée moderne s'était dérobée au dogmatisme théologique en se sécularisant ${ }^{35}$, il nous advint d'étudier comment la science expérimentale s'était affranchie de l'empire d'Aristote. [Rougier 2010, 46]

C'est ce qu'affirment tout aussi nettement les premières lignes de l'article du Mercure de France :

Ayant consacré un volumineux ouvrage à l'origine de l'évolution de la Scolastique latine, il m'advint de chercher comment elle avait décliné, puis succombé au XVII ${ }^{\mathrm{e}}$ siècle. [Rougier 1931b, 513]

Ainsi les liens entre les deux textes sont très étroits, et cela d'abord parce qu'ils sont tous deux des textes foncièrement anti-thomistes ou anti-scolastique, ce qui pour Rougier est à peu près tout un : il s'agit dans le premier de dresser le constat de la «faillite philosophique » de la scolastique, et dans le second d'étudier comment la science moderne s'est construite en se libérant du péripatétisme.

\section{1 - Le renouveau du thomisme}

Ce qui pose d'emblée une première question, car une telle ambition peut paraître aujourd'hui bien surprenante : pourquoi, dans les années 1920, alors que sur le plan philosophique, domine largement en France le spiritualisme des quatre « $\mathrm{B} »$ : Brunschvicg, Boutroux, Bergson, Blondel, et que sur le plan scientifique le combat contre l'aristotélisme scolastique appartient, estime-t-on habituellement, largement au passé, s'obstiner à ferrailler contre un adversaire depuis longtemps vaincu ${ }^{36}$ ? C'est évidemment négliger que, même si l'aristotélisme a cessé de fournir la base de l'enseignement dans les universités et d'apparaître à la très grande majorité des savants comme une alternative possible aux théories construites depuis le XVII ${ }^{\mathrm{e}}$ siècle, il n'a cependant jamais cessé d'être enseigné dans

35. [Rougier 1925a, Livre IV, chap. ${ }^{\mathrm{er}}$ ].

36. C'est implicitement le point de vue de Koyré qui, dans le fond, ne juge pas absolument indispensable un tel travail, même s'il a pris la peine d'en publier une très longue recension [Koyré 1926]. 
les établissements religieux catholiques. Surtout, c'est négliger ou ignorer l'importante offensive menée par le Vatican à la fin du XIX ${ }^{\mathrm{e}}$ siècle pour impulser un mouvement de retour à Thomas d'Aquin, pour, comme le dit l'Encyclique Aeterni Patris, «remettre en vigueur l'admirable doctrine de saint Thomas d'Aquin », et « rendre à [son] enseignement son ancien lustre ». Et cela tout à la fois contre le progrès des sciences, du positivisme, de son dérivé le scientisme, voire du matérialisme autant que du républicanisme dont ce progrès était jugé inséparable. Toutefois, plutôt que de tourner le dos au progrès scientifique, perspective largement irréaliste, l'Encyclique propose de le contrôler en restaurant l'ancienne alliance de la science et de la métaphysique. Le retour au « docteur angélique » sera ainsi bénéfique non seulement à la foi catholique et à la société tout entière, mais il est également jugé souhaitable pour l'avancement de toutes les sciences, notamment des sciences physiques qui ne pourront que gagner à cette réconciliation avec la métaphysique :

Aussi, les sciences physiques elles-mêmes, si appréciées à cette heure, et qui, illustrées de tant de découvertes, provoquent de toute part une admiration sans bornes, ces sciences, loin d'y perdre, gagneraient singulièrement à une restauration de l'ancienne philosophie. Ce n'est point assez pour féconder leur étude et assurer leur avancement, que de se borner à l'observation des faits et à la contemplation de la nature; mais les faits constatés, il faut s'élever plus haut, et s'appliquer avec soin à reconnaître la nature des choses corporelles et à rechercher les lois auxquelles elles obéissent, ainsi que les principes d'où elles découlent et l'ordre qu'elles ont entre elles, et l'unité dans leur variété, et leur mutuelle affinité dans la diversité. On ne peut s'imaginer combien la philosophie scolastique, sagement enseignée, apporterait à ces recherches de force, de lumière et de secours.

Simultanément, l'Encyclique se propose de combattre le préjugé qui voudrait que le thomisme en particulier et la scolastique en général aient été un obstacle au progrès des sciences :

À ce propos, il importe de prémunir les esprits contre la souveraine injustice que l'on fait à cette philosophie, en l'accusant de mettre obstacle au progrès et au développement des sciences naturelles. [...] Saint Thomas, le bienheureux Albert le Grand, et d'autres princes de la scolastique, ne s'absorbèrent pas tellement dans la contemplation 
de la philosophie, qu'ils n'aient aussi apporté un grand soin à la connaissance des choses naturelles; bien plus, dans cet ordre de connaissances, il est plus d'une de leurs affirmations, plus d'un de leurs principes, que les maitres actuels approuvent, et dont ils reconnaissent la justesse. En outre, à notre époque même, plusieurs illustres maîtres des sciences physiques attestent publiquement et ouvertement que, entre les conclusions admises et certaines de la physique moderne et les principes philosophiques de l'école, il n'existe en réalité aucune contradiction.

Les effets de cette politique sont bien connus. Pour s'en tenir au cas de la France, on assiste à un renouveau des études de philosophie médiévale, illustrées notamment par les noms de Jacques Maritain et d'Étienne Gilson. Dans le domaine de la physique et de l'épistémologie, les travaux de Pierre Duhem s'efforcent de prouver qu'il n'y a, il est vrai, aucune contradiction entre les théories physiques les plus récentes et «les principes philosophiques de l'École». Bien au contraire. Les succès de la thermodynamique révèlent en effet selon le physicien les insuffisances manifestes du mécanisme, un mécanisme qui a eu pour conséquence de procéder à l'éviction des «qualités» du monde de la physique. Il propose donc de réintroduire dans la physique ces qualités qui en ont été abusivement chassées. On comprend que certains commentateurs, comme Abel Rey, aient immédiatement perçu et souligné « la proximité de la physique de Duhem et celle d'Aristote » [Rey 1904, $700]^{37}$. Selon Abel Rey, la mécanique fondée sur la thermodynamique, telle que la conçoit Duhem, constitue un «retour [...] aux principes les plus profonds des doctrines péripatéticiennes », [Rey 1904, 734]. Ou encore un « retour à la scolastique », [Rey 1904, 741 $\left.{ }^{38}\right]$. En outre, en affichant ainsi la volonté de revenir à une physique qualitative, tout en professant, malgré ses dénégations, un «scepticisme scientifique intégral » Duhem a, toujours selon Abel Rey, professé la «philosophie scientifique d'un croyant » [Rey 1904, 744]. En réponse à cette accusation, qu'il récuse entièrement, si on l'entend au sens fort selon lequel ses positions

37. Émile Meyerson a procédé au même constat : Duhem voulait « orienter la physique vers un retour au péripatétisme»[Meyerson 1908, 96] ou encore : «le retour au péripatétisme, préconisé avec tant de force et de savoir par Duhem nous paraît impossible », [Meyerson 1908, 480]. Meyerson précise cependant que Duhem vers la fin de sa vie a quelque peu changé d'opinion, ce que nous allons constater.

38. C'est ainsi à plusieurs reprises qu'Abel Rey souligne la volonté affichée par Pierre Duhem de faire retour à Aristote et de marquer sur de nombreux points l'accord foncier des théories d'Aristote et celles des thermodynamiciens du début du xx ${ }^{\mathrm{e}}$ siècle, [Rey 1904, 734, 738]. 
religieuses auraient commandé ses positions scientifiques, Duhem revendique néanmoins hautement sa qualité de catholique, et se réclame de la position défendue par Léon XIII, estimant à son tour que la philosophie scolastique a rendu et ne cessera de rendre de grands services à la science [Duhem 1905b, 44; 133].

Le combat mené par Rougier contre la scolastique et contre le thomisme, était donc bien loin d'être, lorsqu'il l'entame, un combat d'arrière-garde. Il était au contraire urgent, à ses yeux évidemment, face à l'importance que revêtait alors le renouveau du thomisme, sur le plan philosophique comme sur le plan scientifique, de le combattre. D'autant que, loin de minimiser l'adversaire, Rougier reconnaissait dans l'Avertissement de De Torricelli à Pascal que le système de l'École «constitue un appareil d'une grandeur et d'une cohésion admirables », et voyait dans les grandes qualités de ce système l'explication du fait que « ceux de nos contemporains qui prêchent le retour à la Scolastique paraissent s'en accommoder encore parfaitement de nos jours » [Rougier 2010, 46]. Il devenait donc tout à fait nécessaire d'en révéler aussi les défauts.

\section{2 - « Le thomisme et la critique sympathique de M. Gilson »}

On comprend mieux également du coup les différents liens qui se sont noués entre une série de textes de Rougier parus à peu près au même moment, dont un article publié lui aussi dans le Mercure de France, dans la livraison précédant celle où Rougier dénonce la méthode de M. Brunschvicg. Ce premier article est tout aussi polémique que le sera le suivant. Intitulé « Le thomisme et la critique sympathique de M. Gilson », il constitue ce que l'on hésite à considérer comme la recension, tant sa date de parution est tardive, de l'ouvrage de Gilson [Gilson 1919]. Plus de huit ans plus tard! Il constitue donc plutôt une riposte aux critiques dont La Scolastique et le Thomisme, paru entre temps, a fait l'objet ${ }^{39}$. C'est un article d'humeur, au même titre que celui qui suivra et qui prendra Brunschvicg pour cible.

Il offre d'abord l'occasion de constater la présence de ces réemplois dont Rougier est coutumier, réemplois qui tissent des liens entre plusieurs de ses textes. Des réemplois qui peuvent certes paraître anecdotiques. Ainsi est-il question dans l'article dirigé contre Gilson du « Maure hérétique, Averroès », introducteur d'Aristote dans la scolastique latine.

39. Sur la réception de La Scolastique et le Thomisme, voir Jacques Courcier [Pont \& Padovani 2006]. 
Dans De Torricelli à Pascal, il parle du «philosophe grec, patronné dans l'Occident latin par un Maure, réputé grand blasphémateur et prince des mécréants, Averroès » [Rougier 2010, 46]. Mais cette formule est ellemême reprise de La Scolastique et le Thomisme, où il est était déjà question d'un «Maure à turban, réputé grand blasphémateur et prince des mécréants ». Toujours dans ce même article, où Rougier commence par tourner en dérision la «méthode de sympathie » prônée par Bergson ${ }^{40}$ et mise en œuvre par le philosophe médiéviste, il saisit l'occasion de réutiliser ses récentes recherches sur Pascal :

Étudiez-vous Blaise Pascal? Il ne vous suffira pas, pour trancher son conflit avec Descartes sur la priorité de l'expérience du Puy-de-Dôme, de compulser toutes les pièces du procès. Il faudra donc se mettre à genoux devant son masque, l'invoquer et entrer en transes. La méthode de sympathie participe de l'effusion lyrique, de l'infusion de la grâce, du ravissement et de l'extase. [Rougier 1931a, 341-342 ${ }^{41}$ ]

Ce passage vise en même temps explicitement Émile Boutroux, l'un des premiers à avoir pratiqué cette méthode, et cela très précisément dans un ouvrage consacré à Pascal ${ }^{42}$. Émile Boutroux qui a également participé à la publication des Euvres de Pascal avec Léon Brunschvicg. Ce dernier réemploi prouve que Rougier, s'il ne l'a pas publié, a tâché de réutiliser au maximum son manuscrit. Mais ce réemploi est ici plus que stylistique : il vise, à travers Bergson, à travers Boutroux, le spiritualisme universitaire, qui faisait figure de pensée officielle et contre lequel ferraille également Rougier : nous reviendrons sur ce point.

Pour l'instant, revenons à la question des liens qui rattachent $D e$ Torricelli à Pascal à La Scolastique et le Thomisme et voyons maintenant sur le fond comment le premier constitue lui aussi une pièce dans le dispositif de lutte de Rougier contre le néo-thomisme. Le propre de la scolastique et du thomisme, que Rougier identifie largement, est, assure-t-il,

40. Rougier la définit comme une méthode qui consiste à sympathiser avec l'objet à étudier et qui fait de cet acte l'acte même de la connaissance.

41. On ne peut évidemment que rapprocher ces formules de celles que l'on lit dans [Rougier 2010, 232] notamment.

42. «Pascal, avant d'écrire, se mettait à genoux et priait l'Être infini de se soumettre tout ce qui était en lui, en sorte que cette force s'accordât avec cette bassesse. Par les humiliations, il s'offrait aux inspirations. Il semble que celui qui veut connaître un si haut et si rare génie dans son essence véritable doive suivre une méthode analogue, et, tout en usant, selon ses forces, de l'érudition, de l'analyse et de la critique, qui sont nos instruments naturels, chercher, dans un docile abandon à l'influence de Pascal lui-même, la grâce inspiratrice qui seule peut donner à nos efforts la direction et l'efficace », [Boutroux 1900, non paginé]. 
d'avoir tenté la synthèse de deux dogmatismes, la synthèse d'un dogmatisme religieux et d'un dogmatisme profane, la synthèse du christianisme et de l'aristotélisme, la synthèse de la raison et de la foi, et cela au prix d'une subordination de la logique et de l'ontologie aristotéliciennes à la dogmatique chrétienne, c'est-à-dire à la théologie.

La Scolastique et le Thomisme est essentiellement destiné à mettre en évidence l'échec de cette entreprise. Une telle conciliation exigeait en effet de transformer la distinction de l'essence et de l'existence, qui est pour Rougier une distinction purement logique, purement formelle, en une distinction réelle : seul ce « réalisme» ontologique permettait de «christianiser » Aristote, c'est-à-dire d'échapper au panthéisme, de prouver l'existence de Dieu par la contingence du monde, d'affirmer l'immortalité de l'âme, etc. Cette tentative de conciliation, ne cesse de répéter Rougier, a été un échec, et toute La Scolastique et le Thomisme est une longue « démonstration» de cet échec ${ }^{43}$.

Cet échec est d'abord et avant tout un échec philosophique, rendu tangible par les contradictions auxquelles Thomas d'Aquin est acculé et par les incohérences auxquelles il est contraint, quand il est obligé d'aller chercher chez Avicenne, mais aussi chez bien d'autres philosophes, les éléments nécessaires à son argumentation. Rougier emprunte sa conclusion à Pierre Duhem : le thomisme est une «marqueterie », composée d'une «multitude de pièces empruntées à toutes les philosophies ». Il n'est donc pas « une doctrine, il est une aspiration et une tendance; il n'est pas une synthèse, mais un désir de synthèse », [Rougier 1931a, $370^{44}$. Et si l'on tient à ce qu'il soit une doctrine, alors le thomisme est une « doctrine éclectique et incohérente », [Rougier 1931a, 370].

Thomas d'Aquin n'est donc aucunement, comme l'a prétendu l'Encyclique Aeterni Patris de Léon XIII et comme le prétendent les néothomistes, le «premier philosophe moderne ». Le premier philosophe moderne est bien plutôt celui qui a rejeté le réalisme ontologique d'Aristote, Guillaume d'Ockham. La Scolastique et le Thomisme peut ainsi logiquement se clore par différents développements dans lesquels Rougier analyse rapidement la façon dont la philosophie occidentale s'est « affranchie du joug de la scolastique », et cela sous plusieurs aspects, dont celui de la sécularisation progressive des sciences de la nature. Loin que l'aristotélisme ou le péripatétisme aient fourni les fondements d'une véritable philosophie de la nature, celle-ci n'a pu se constituer qu'en s'af-

43. Sur cet ouvrage, lire Laurent Cesalli, «Louis Rougier et la "mentalité réaliste" », ainsi qu'Alain de Libera, «La scolastique : une faillite? Louis Rougier, historien in partibus », [Pont \& Padovani 2006, 157-175; 177-206].

44. Le texte de Duhem cité par Rougier se trouve dans [Duhem 1954, V, 576]. 
franchissant d'Aristote comme l'ont fait à partir du XIV siècle, et à la suite de Guillaume d'Ockam, « les maîtres nominalistes de l'Université de Paris », c'est-à-dire cette école parisienne de physique illustrée par les travaux d'Oresme et de Buridan sur le mouvement et la théorie de l'impetus [Rougier 1925a, Livre IV].

Or c'est très précisément par l'évocation de ce même processus d'affranchissement tel qu'il a été accompli dans le domaine des sciences que s'ouvre l'Avertissement de De Torricelli à Pascal : ainsi celui-ci commence où finissait La Scolastique et le Thomisme. Quand Rougier présente De Torricelli à Pascal comme la suite ou le prolongement de La Scolastique et le Thomisme, il faut par conséquent entendre cette formule au sens fort : non seulement il fait chronologiquement suite à $L a S c o-$ lastique et le Thomisme, non seulement Rougier y poursuit son combat contre le thomisme, mais il reprend l'étude du processus d'affranchissement des sciences de la nature vis-à-vis de l'aristotélisme là où il l'avait interrompue, après Galilée et ses travaux sur la dynamique. Résumant à grands traits ce processus historique de libération de la pensée scientifique à l'égard de la physique d'Aristote dont il a déjà esquissé le récit dans La Scolastique et le Thomisme, Rougier en retient quelques noms et quelques moments essentiels : d'abord le nom de Guillaume d'Ockham, qu'il présente à nouveau comme l'initiateur de ce processus, puis les noms de Copernic, de Kepler, de Galilée, dont la dynamique signe le triomphe de la physique moderne sur la physique de l'École. Un triomphe cependant incomplet : d'autres batailles restent à mener. Les questions de pneumatique et d'hydrostatique, telles qu'elles se sont posées dans la première moitié du XVII ${ }^{\mathrm{e}}$ siècle avec Stevin, Baliani, Torricelli, Roberval, et enfin Pascal, constituent le nouveau champ de bataille contre « la science du Stagirite ». Un champ de bataille où l'on va assister, notamment à l'occasion de l'expérience du puy de Dôme, à « la déroute définitive de la physique péripatéticienne, de la physique oppressive de la Scolastique » : ce sont les derniers mots de sa Conclusion.

\section{3 - De Torricelli à Pascal et le combat contre le péripatétisme}

On comprend mieux pourquoi Rougier avait primitivement baptisé son manuscrit La Déroute de la scolastique. C'est cette déroute qui constituait selon lui l'enjeu principal des travaux conduits par les savants italiens puis français sur la question du vide et de la pression atmosphérique. C'est aussi cette déroute annoncée qui explique toujours selon lui l'âpreté de la controverse qui a opposé alors plénistes et vacuistes, tenants de la 
force attractive du vide et partisans de la colonne d'air; qui explique autant la diversité et la complexité des positions adoptées que les revirements d'opinion et les volte-face : Descartes est pléniste mais favorable à la « colonne d'air », Roberval est d'abord vacuiste et partisan de « la force attractive du vide», puis se rallie au plein et à la colonne d'air, Pascal ne se soucie d'abord que du vide et ne pose que tardivement la question de la «pesanteur et pression de l'air », Mersenne quant à lui change plusieurs fois d'opinion. Ces hésitations et ces théories concurrentes s'expliquent aisément selon Rougier si l'on mesure qu'à travers ces questions, il ne s'agissait de rien de moins que de construire «l'édifice classique de l'hydrostatique » sur un « champ de décombres gothiques » [Rougier 2010, 48]. S'expliquent enfin aussi aisément l'exaspération des revendications de priorité qui, dans les années 1647-1648 ont déchiré la communauté savante, et les divergences de points de vue entre les analystes modernes de la querelle, qui, nous l'avons déjà signalé, s'ils sont presque tous favorables à Pascal, ne le sont pas cependant tous, ni également, ni pour les mêmes raisons, des divergences que Rougier résume rapidement : on peut ainsi vérifier qu'il était devenu à peu près impossible de parler des travaux de Torricelli et de Pascal sans à tout le moins évoquer le débat déclenché par Félix Mathieu.

On comprend surtout pourquoi Rougier s'est fait historien de la physique; c'est à l'évidence d'abord et avant tout pour poursuivre son histoire de la philosophie ou de la pensée occidentale commencée avec La Scolastique et le Thomisme et pour en renforcer les conclusions polémiques. Après avoir montré comment la pensée occidentale s'est affranchie de la cosmologie aristotélicienne avec Copernic puis de la dynamique aristotélicienne avec Galilée, il s'agit de montrer comment ce processus s'est poursuivi en se concentrant sur le terrain de la statique des fluides, avec les travaux de Torricelli et de Pascal notamment. Rougier montre ainsi en détail et longuement - il y consacre une partie de l'Avertissement, y revient à plusieurs reprises dans le corps de l'ouvrage et enfin y revient assez longuement dans la Conclusion - , comment les théories professées sur l'existence du vide, de la pesanteur et pression de l'air, puis de l'élasticité des gaz « démentaient violemment les principes fondamentaux de la logique, de l'ontologie et de la physique de l'École » [Rougier $2010,48]$. Le premier chapitre de l'ouvrage, qui s'intitule de façon significative «L'expérience de Torricelli et la scolastique », est spécialement destiné à expliciter cette triple remise en question. De l'ontologie d'Aristote en premier lieu, car poser l'existence du vide, c'est admettre l'existence d'un non-être, ce qui constitue une contradiction insurmontable. Ensuite de la logique et de la table des catégories d'Aristote, qui professe 
que toute chose est nécessairement ou substance ou accident. Or le vide n'est ni substance ni accident, car toute substance est soit matérielle, soit spirituelle. Mais l'espace vide n'est ni corps ni esprit, il n'est donc pas une substance; il n'est pas davantage un accident, car tout accident est accident d'une substance, et l'on ne voit pas de quelle substance le vide serait l'accident. Enfin de la physique d'Aristote, et cela de plusieurs façons. D'après le Stagirite, la vitesse d'un corps en mouvement dépend non seulement de la force avec laquelle il est mu initialement, mais aussi de la résistance que lui oppose le milieu dans lequel il se meut. Or dans le vide, ce mouvement ne rencontre aucune résistance : il devrait par conséquent atteindre une vitesse infinie, proprement inconcevable selon Aristote. Parler par ailleurs de la pesanteur de l'air, c'est affirmer que l'air est pesant. Or dans la physique des éléments d'Aristote, l'air est léger, non pas relativement mais absolument, ce qui signifie qu'il est dénué de poids ${ }^{45}$. Comme par ailleurs, les éléments ne pèsent pas en eux-mêmes, c'est-à-dire dans leur lieu naturel, l'air ne pèse pas dans l'air, ni l'eau dans l'eau et aucune pression ne saurait s'exercer en eux (« dans une masse fluide $»$, traduit Rougier). Par ailleurs encore, l'affirmation de ce que Rougier n'hésite pas non plus à désigner comme l'élasticité des gaz ${ }^{46}$, et que l'on appelle dans les textes de l'époque la «raréfaction de l'air », c'est-à-dire le fait que l'air se dilate spontanément pour occuper tout l'espace disponible, ce que Roberval a établi en 1648, contredit le dogme aristotélicien de la passivité des substances inanimées, c'est-à-dire leur incapacité à se donner elles-mêmes un mouvement. À tout mouvement, enseigne en effet également Aristote, il faut un moteur. La raréfaction spontanée des gaz, de l'air est donc elle aussi impensable dans le cadre de la physique aristotélicienne.

Que les expériences réalisées dans les années 1647-1648 aient constitué par ailleurs, dans l'histoire de la pensée occidentale, une innovation méthodologique importante, en détachant la pensée scientifique du culte des Anciens, de l'esprit de système, des chimères de la spéculation a priori et fondé un scepticisme raisonnable tout en inaugurant la physique expérimentale, Rougier ne manque pas de le signaler; mais cela lui importe cependant à l'évidence beaucoup moins que le fait que la statique des fluides ait ruiné « la logique, la méthode et l'ontologie d'Aristote ». Ainsi, et même si Louis Rougier traite bien de l'histoire de la physique au XVII ${ }^{\text {e }}$ siècle, et de l'épisode fondateur de la statique des

45. Il faut ici distinguer la position d'Aristote et celle des aristotéliciens. Aristote a tenté de mesurer le poids de l'air, en pesant une outre gonflée d'air. Ce sont les aristotéliciens qui ont ultérieurement professé une opinion différente. Voir à ce sujet [Adam 1888].

46. Nous reviendrons sur ces anachronismes dont Rougier est coutumier. 
fluides, ce texte reste cependant, au moins dans les intentions de son auteur, un texte foncièrement anti-thomiste ou un texte anti-scolastique. Et c'est autant contre la scolastique médiévale que contre les néo-thomistes contemporains que combat Rougier ${ }^{47}$.

\section{4 - Rougier et Duhem}

Ce combat a donc commencé avec La Scolastique et le Thomisme. L'ouvrage a été mal reçu, par les néo-thomistes justement, ce qui était sans aucun doute prévisible ${ }^{48}$. Si des critiques de fond lui ont été adressées, Rougier a été aussi accusé d'avoir amplement utilisé les travaux de Pierre Duhem, sans le signaler. On peut donc supposer que la virulence des attaques de Rougier contre Gilson dans le Mercure de France s'explique largement par cette circonstance. Ce qui confirme que dans les deux articles publiés dans ce périodique, les cibles de Rougier sont des cibles contemporaines et que l'histoire des sciences ou l'histoire de la philosophie ne sont que des prétextes pour régler des questions tout à fait actuelles. Ce qui pose surtout la question des rapports de Rougier avec Duhem, en commençant par l'accusation de plagiat ${ }^{49}$. Sans entrer dans le détail de cette accusation, on constate d'une part que Rougier rend à plusieurs reprises un hommage appuyé à Duhem. Dès la préface de $L a$ Scolastique et le Thomisme, Rougier adopte ouvertement une des thèses maîtresses de Duhem :

En tant que philosophie de la nature, l'Aristotélisme a complètement échoué. Dès le XIV e siècle, comme l'ont établi les recherches de Pierre Duhem, la mécanique, la cosmologie, la physique du Lycée sont battues en brèche par les Maîtres nominalistes de l'Université de Paris. [Rougier 1925a, XX]

47. C'est en cette façon essentiellement que De Torricelli à Pascal s'insère dans l'œuvre de Rougier, et par-delà La Scolastique et le Thomisme, peut être rattaché notamment aux Paralogismes du rationalisme, [Rougier 1920] ou à des ouvrages plus tardifs comme le Traité de la connaissance, [Rougier 1955]. Il est difficile en effet de trouver dans sa pratique de l'histoire des sciences un écho de ses positions épistémologiques, comme l'on pourrait s'y attendre. Nous n'avons donc pas tenté d'opérer de ce point de vue des rapprochements que rien dans le texte de Rougier ne justifie.

48. Voir Jacques Courcier dans [Pont \& Padovani 2006].

49. À ce sujet, voir Jacques Courcier [Pont \& Padovani 2006]. Selon Jacques Courcier, Rougier a été accusé d'avoir amplement emprunté non seulement à Duhem, mais à bien d'autres auteurs. En ce qui concerne Duhem, Rougier aurait utilisé les volumes inédits du Système du monde, notamment le volume V, que Duhem l'aurait chargé de publier, ce que dément la fille de Duhem. 
Et il lui doit également la thèse de l'incohérence de la doctrine de Thomas d'Aquin, qu'il lui emprunte tout aussi explicitement. Très certainement cependant, ces dettes avouées cachent-elles d'autres dettes, inavouées cette fois. C'est sans doute l'une des raisons pour lesquelles dans « La critique sympathique de M. Gilson», qui est à la fois une réponse et une riposte face aux critiques qu'il a essuyées, Rougier réaffirme à maintes reprises sa double dette à l'égard de Duhem, une façon de ne pas donner l'impression qu'il dissimule celle-ci. Assez logiquement, on voit donc également Rougier, dans l'Avertissement de De Torricelli à Pascal, rendre hommage aux travaux de Duhem pour avoir « reconnu», en des études estimées «mémorables », «l'aurore de cet affranchissement chez les Maîtres nominalistes de la Faculté des arts de l'Université de Paris » : il s'agit bien évidemment de l'affranchissement de la philosophie naturelle par rapport à l'aristotélisme; et il situe explicitement ses propres travaux historiques dans la lignée de ceux de Duhem.

Il faut par ailleurs signaler une autre difficulté, plus importante. Celle que font surgir précisément ces hommages multiples à Duhem dont Rougier n'a cessé de se réclamer, autant dans La Scolastique et le Thomisme que dans l'Avertissement de De Torricelli à Pascal, ou dans l'article du Mercure de France consacré à Gilson. Car, apparemment, beaucoup de choses séparent les deux hommes, à commencer par le catholicisme non seulement avoué, mais hautement proclamé de Duhem, alors que Rougier revendique pour sa part un athéisme résolu. Plus généralement, et cela pouvant être considéré comme la conséquence de ceci, l'appartenance présumée de Duhem au mouvement néo-thomiste contre lequel Rougier bataille aurait dû tout autant contribuer à accroître la distance entre les deux hommes.

On peut difficilement suspecter l'admiration affichée par Rougier à l'égard de Duhem, non seulement pour la raison relativement secondaire que Rougier n'était certainement pas homme à dissimuler ses véritables sentiments, mais aussi parce que l'on peut relever une similitude assez grande entre les interprétations proposées par Rougier de l'histoire du mouvement de la pensée scientifique et celles de Duhem. Rougier et Duhem partagent en effet la même périodisation de l'histoire des sciences, situant tous les deux la naissance de la science moderne ou du moins son aurore au XIV ${ }^{\mathrm{e}}$ siècle. Cette identité de vue évidemment ne doit pas étonner si Rougier a bien emprunté à Duhem sa lecture et surtout cette périodisation. Reste cependant à rendre compte de la prédilection, qui a pu aller jusqu'au plagiat, d'un adversaire résolu du thomisme à l'égard d'un néo-thomiste. Cette convergence et cette prédilection ne font question cependant que si Duhem peut réellement être rangé dans la caté- 
gorie des néo-thomistes; or, la question de l'appartenance de Duhem au mouvement néo-thomiste est très controversée. Et si elle est controversée, c'est justement en partie (ou largement), parce que les positions de Duhem à l'égard d'Aristote, du péripatétisme, de Thomas d'Aquin et de la scolastique sont loin d'être simples et univoques.

Il importe à ce sujet d'opérer plusieurs distinctions, et d'abord une distinction d'ordre chronologique. Les positions de Duhem ont évolué, et cela d'ailleurs en raison même de l'évolution de l'orientation de ses travaux qui sont allés de la physique à l'épistémologie pour se tourner ensuite entièrement vers l'histoire des sciences. La critique duhémienne du mécanisme et son mot d'ordre de retour à Aristote et au péripatétisme correspondent à un moment précis de son œuvre, celui où il est encore plus physicien qu'historien des sciences. On peut se demander en outre quelle était la portée exacte du retour à Aristote que prônait Duhem dans ces années-là. Dans la réponse à Abel Rey que nous avons déjà évoquée, Duhem minimise sérieusement cette portée et se contente de souligner l'analogie de la thermodynamique avec la cosmologie d'Aristote et de l'École, une analogie d'ailleurs assez vague ${ }^{50}$. Pour autant, il récuse entièrement, nous l'avons vu, l'accusation de professer une «physique de croyant », au motif que la cosmologie d'Aristote et celle de la scolastique sont entièrement indépendantes du dogme catholique. Ce qui l'autorise néanmoins à conclure, en accord explicite avec les proclamations de Léon XIII, que cette cosmologie peut servir à développer la science et à conclure également au rôle historiquement positif joué par l'Église catholique sur le développement de la science.

Certes, les travaux d'histoire des sciences entamés par Duhem à partir de 1904 paraissent d'abord conforter les conclusions de ses travaux de physicien et d'épistémologue. C'est dans L'origine de la statique [Duhem 1905] que Duhem apparaît le plus nettement comme l' « inventeur » de la science médiévale, le pourfendeur de l'idée reçue selon laquelle les 《âges théologiques » auraient été un obstacle au développement de la pensée scientifique. C'est dans cet ouvrage qu'il récuse pour la première fois explicitement l'interprétation selon laquelle la science moderne, et notamment cette partie de la science moderne qu'est la statique serait, comme toute la science moderne, née au XVII ${ }^{\mathrm{e}}$ siècle, grâce à Galilée et à Descartes, qu'elle aurait été constituée en rupture avec la statique anté-

50. Cette analogie ne peut être perçue en effet qu'à des conditions fort exigeantes : «si l'on débarrassait la Physique d'Aristote et de la Scolastique du vêtement scientifique usé et démodé qui la recouvre, si l'on faisait apparaître, dans sa vigoureuse et harmonieuse nudité, la chair vive de cette cosmologie, on serait saisi de la ressemblance qu'elle présente avec notre moderne théorie physique », [Duhem 1906c, 471]. 
rieure, notamment avec la statique aristotélicienne, voire avec la statique médiévale, à la condition bien entendu que l'on accepte de reconnaître l'existence d'une telle statique au Moyen Âge, ce à quoi Duhem s'est fortement employé. L'origine de la statique ne remonte pas à Galilée ni à Descartes, mais à un savant dont il redécouvre l'existence, Jordanus de Nemore, qui aurait vécu au XII ${ }^{\mathrm{e}}$ siècle, et qui a sinon explicitement énoncé, mais au moins pressenti les principes de la statique et la loi de la chute des graves. Selon Duhem, toute l'histoire ultérieure de la statique n'a été en quelque sorte que le développement des intuitions de Jordanus de Nemore. De son côté, l'école parisienne de mécanique des $\mathrm{XIV}^{\mathrm{e}}$ et $\mathrm{XV}^{\mathrm{e}}$ siècles, de Buridan à Oresme, a anticipé les principes de la dynamique galiléenne, notamment avec la théorie de «l'impetus », cette «impression» de mouvement qui permet au corps mu de conserver son mouvement une fois séparé de son mobile et dans lequel Duhem veut voir l'anticipation du principe d'inertie. Dans tous ses travaux ultérieurs, dans ce qui deviendra Le Système du monde, Duhem a continué à construire, avec une ampleur inégalée jusqu'alors, l'histoire de la science médiévale.

Toutefois, on ne doit pas négliger à la fois une évolution et une incertitude, ou au moins une hésitation dans la pensée de Duhem, qui a varié dans ses interprétations. Il semble bien en effet qu'au fur et à mesure qu'il développe ses travaux sur cette science médiévale, il prenne progressivement et de plus en plus ses distances vis-à-vis du péripatétisme, de Thomas d'Aquin et de la scolastique ${ }^{51}$. D'autre part, s'il a bien fait remonter la date de naissance de la science moderne très en amont du XVII ${ }^{\mathrm{e}}$ siècle, il a hésité en ce qui concerne la détermination de cette date. Les condamnations d'Étienne Templier, prononcées en 1277 et qui visent des thèses aristotéliciennes [Duhem 1909, 412], [Duhem 1954, 66], [Duhem 1956, 7-8] ou l'école parisienne de Buridan qui, au $\mathrm{XIV}^{\mathrm{e}}$ siècle, posent les bases d'une physique et d'une dynamique non

51. Alexandre Koyré propose de cette évolution de la pensée de Duhem une explication très particulière. Selon lui, l'aristotélisme et le thomisme, en tant que philosophies de la nature, sont définitivement périmés et ils ne peuvent être ressuscités.

Personne d'ailleurs parmi les thomistes, poursuit-il, n'a tenté cette aventure sauf Duhem, qui ayant cherché, et n'ayant pas réussi à refaire une physique aristotélisante en a, par dépit, conçu une aversion violente et injuste - envers saint Thomas.

Koyré propose cette explication de la désaffection de Duhem à l'égard de Thomas d'Aquin dans sa recension de La Scolastique et le Thomisme, [Rougier 1925a]. Cette longue recension est plutôt favorable à l'ouvrage, même si elle n'est pas exempte de critiques, celle notamment que nous avons signalée. En tout cas, Koyré a lu attentivement Rougier. 
aristotélicienne [Duhem 1913, IX], [Duhem 1956, 340]. Peu importe en ce qui nous concerne, on soulignera simplement que, quelle que soit la réponse que Duhem donne finalement à cette question, dans les deux cas, ce n'est jamais dans le thomisme mais hors du thomisme ou contre lui que Duhem repère l'impulsion qui a donné naissance à la science moderne. En même temps, Duhem n'a pas hésité à décrire le processus de destruction progressive de l'édifice de la scolastique. En revanche, dans les deux cas, Duhem insiste sur le fait que c'est bien de l'Église catholique qu'est venue cette impulsion nécessaire au développement de la science moderne. En résumé, catholique certes, mais non thomiste : sur cette base, celle d'un rejet du thomisme, un accord était évidemment possible entre Rougier et Duhem, et de fait, Rougier ne retient de l'œuvre de Duhem que l'étude de ce processus d'affranchissement de la science moderne vis-à-vis de l'École et du péripatétisme, dont il désigne, nous l'avons dit, les mêmes noms des acteurs principaux. Et le catholicisme de Duhem, «dont, souligne-t-il, on ne saurait soupçonner le parti $\operatorname{pris}^{52} »$, sert ainsi essentiellement à Rougier à cautionner sa thèse de l'incohérence de Thomas d'Aquin, thèse d'ailleurs, nous l'avons dit, qu'il lui emprunte. Il pouvait difficilement condamner un catholicisme qui lui était en la circonstance si utile! Enfin, on ne saurait ignorer la proximité, sur le plan politique, de Duhem et de Rougier, tous les deux hommes de droite, hostiles au républicanisme et à l'héritage idéologique de la Révolution française.

\section{4 - « L'affaire Pascal »}

Si le combat mené par les savants du XVII ${ }^{\mathrm{e}}$ siècle contre le péripatétisme importe ainsi prioritairement à Rougier, il n'en a pas moins accordé dans son manuscrit une place non négligeable à la controverse suscitée par la publication des articles de Félix Mathieu. On peut certes en trouver l'explication dans le fait que l'ampleur du débat avait été telle qu'il était difficile de paraître l'ignorer ${ }^{53}$. On peut aussi tenter d'expliquer autrement l'importance que Rougier lui attache.

52. Préface, p. XXVII. Même remarque dans [Rougier 1931a, 370], à propos de l'incohérence du thomisme :

C'est la conclusion à laquelle s'est trouvé aboutir un homme qui n'est pas suspect de partialité, un catholique fervent, Pierre Duhem, au terme de la longue étude qu'il a consacrée au thomisme.

53. Seul Cornelis de Waard [Waard 1936] paraît ignorer l'existence même de cette querelle. 


\section{1 - La position de Rougier dans « l'affaire Pascal »}

De cette controverse, il est question dès l'Avertissement, à plusieurs reprises dans le texte lui-même, notamment dans le chapitre VI intitulé «Le réquisitoire de M. Mathieu » et enfin assez longuement en conclusion. Il n'est pas facile de résumer la position exacte de Rougier en ce qui concerne 《l'affaire Pascal »: il faudrait déjà être capable d'exposer rapidement, en quelques mots ou en quelques phrases la longue argumentation de Félix Mathieu, ainsi que celle de ses adversaires. Tout au plus peut-on tenter de clarifier le débat en le réduisant à trois questions principales. La première question que posait Félix Mathieu était celle de la priorité de Pascal en ce qui concerne l'invention de deux expériences : celles du vide dans le vide et celle du puy de Dôme. La seconde question était celle du «faux» rédigé par Pascal pour s'attribuer indûment la paternité de ces deux expériences : c'est l'accusation d'avoir antidaté la lettre à Florin Périer du 19 septembre 1647. La troisième question, que pose plus spécifiquement Rougier — pour Félix Mathieu la réponse va de soi - est celle de la «sincérité » de Pascal. On soulignera que Rougier tient à affirmer son indépendance à l'égard de Félix Mathieu : son rôle, assure-t-il, «n'est ni de le disculper, ni de l'accuser ${ }^{54} »$ et à plusieurs reprises, Rougier prend en effet ses distances à l'égard des positions défendues par l'homme de lettres.

En ce qui concerne la première question, Rougier à l'évidence n'est nullement convaincu de la priorité de Pascal, et quand bien même il s'écarte des positions de Félix Mathieu sur des points de détail, il le rejoint sur deux de ses principales affirmations : l'expérience du vide dans le vide a été réalisée pour la première fois par Auzoult, ainsi qu'en témoigne la Dissertatio anatomica de circulatione sanguinis et chyli motu [Pecquet 1651] et c'est Descartes qui a suggéré à Pascal l'idée d'accomplir l'expérience ordinaire du vide à des altitudes variées. Plus généralement, Rougier est convaincu que Pascal à plusieurs reprises a présenté comme réellement effectuées des expériences imaginaires, des « expériences de pensée $^{55} »$. À la différence de Félix Mathieu, il refuse cependant de «crier au plagiat », dans la mesure où la question de la priorité de Pascal lui importe finalement assez peu. Pour une raison qui, une fois de plus, le

54. Rougier a rencontré Félix Mathieu, qu'il décrit comme «un remarquable érudit, un aristarque doué d'une ironie mordante, un écrivain de race possédant l'art de faire vivre ses personnages », [Rougier 2010, 232]. On peut également s'étonner de cette louange à l'égard d'un homme dont il ne partageait certainement pas les convictions politiques. Mais être victime de Brunschvicg créait sans doute à ses yeux une réelle complicité avec un homme dont la verve de polémiste a pu aussi le séduire.

55. Ce qui était relativement banal au XVII ${ }^{\mathrm{e}}$ siècle. 
rapproche de Duhem. Celui-ci fait partie en effet de ceux qui sont intervenus, dès la parution des articles de Félix Mathieu, dans le débat [Duhem 1906a], sans être d'ailleurs autrement choqué par la diatribe lancée contre Pascal. Peu soucieux de polémiquer, Duhem se prononce cependant quant à lui en faveur de Mersenne ${ }^{56}$, dont il affirme qu'il est le premier à avoir proposé d'effectuer l'expérience ordinaire du vide à des altitudes différentes dans un texte imprimé, la précision est importante. En ce qui concerne la priorité de l'idée proprement dite de cette expérience, il considère la question comme indécidable, au nom de l'argument selon lequel quand une découverte est « dans l'air du temps », il est inévitable que plusieurs personnes simultanément l'effectuent [Duhem 1906a, 774]. Cette position de Duhem est en fait la conséquence logique de sa conception continuiste de l'histoire des sciences, qui tend à minimiser le rôle des « grands hommes » dans l'histoire des sciences, au profit de la restitution d'un processus conçu comme le résultat d'une multitude d'interventions qui finissent par produire une découverte, longuement préparée. Les querelles de priorité lui paraissent par conséquent sans objet et sans intérêt, et Rougier partage ce point de vue, qu'il lui emprunte sans doute, une fois encore et sous l'autorité duquel il s'abrite ${ }^{57}$.

En ce qui concerne la seconde question, si Rougier paraît à nouveau plutôt disposé à suivre l'argumentation de Félix Mathieu, si, dit-il, « les raisons capitales de soupçonner Pascal, alléguées par M. Mathieu, restent entières »[Rougier 2010, 232], et s'il est tout disposé à considérer la lettre adressée par Pascal à Florin Périer comme « une justification fictive, inventée après coup », il ne se prononce pas catégoriquement sur le caractère véritablement fictif de la lettre de Pascal à Florin Périer : même s'il le tient pour hautement probable, il le considère en même temps comme impossible à prouver et recommande donc la prudence. Le

56. «Dont personne n'eut jamais cure », précise Rougier [Rougier 2010, 48] qui prend ainsi tout de même de temps en temps ses distances à l'égard des positions de Duhem. Il est vrai que même si Mersenne peut sembler avoir conçu l'idée de cette expérience, — c'est l'impression qui résulte de la lecture de ses ouvrages — son attitude est en même temps si hésitante et ses positions théoriques si dépendantes de l'influence ponctuellement exercée par l'un ou l'autre de ses interlocuteurs que la question de la priorité a toujours essentiellement concerné Descartes et Pascal.

57. Voir la longue citation qu'il fait de l'article de Duhem dans la Conclusion de De Torricelli à Pascal. On peut en déduire que Rougier partage également ce continuisme, perceptible dans le titre du chapitre III de De Torricelli à Pascal, chapitre qui s'intitule « Précurseurs et émules de Torricelli ». Ce titre fait inévitablement penser au titre de l'ouvrage de Duhem : Léonard de Vinci. Ceux qu'il a lus, ceux qui l'ont lu, [Duhem 1906b]. Il n'est donc sans doute pas excessif de parler de «fascination» de Rougier pour Duhem, comme le fait Jacques Courcier [Pont \& Padovani 2006]. 
procès introduit par Félix Mathieu lui semble en fait sur ce point sans 《solution définitive» [Rougier 2010, 233].

La question majeure que pose à ses yeux l'épisode dont il reconstruit l'histoire est bien plutôt, à travers lui, celle de la «sincérité de Pascal », dont il dresse, sur le plan psychologique, un portrait peu flatté : « génie hyperbolique » et «impatient de pousser sa gloire», avide de renommée, 《 irascible et vaniteux enfant », coupable de «surenchère orgueilleuse ». Refusant de céder aux prestiges de «l'apologétique édifiante », et «d'entrer en transe devant le masque de Pascal $^{58} »$, - il s'adresse évidemment aux pascaliens zélés - il conclut à son «insincérité ». Pascal est donc convaincu de «mensonges », ceux, notamment, qui grèvent lourdement la Lettre à $M$. de Ribeyre ${ }^{59}$.

Toutefois, et sur ce point il se sépare nettement de Félix Mathieu, qui minimisait sérieusement la portée et l'originalité des travaux scientifiques de Pascal, Rougier reconnaît sans réserves son « génie», qui éclate dans ses traités posthumes, et auquel il consacre un chapitre entier. Sa dette à l'égard de Duhem est une fois de plus importante, et Rougier le cite très longuement. Pascal, dans le Traité de l'équilibre des liqueurs, comme l'a donc montré Duhem, n'a énoncé aucune proposition nouvelle, mais il a su ranger les propositions éparses et sans lien qu'il a empruntées à Mersenne, à Stevin, à Benedetti, à Galilée, à Torricelli et à Descartes, selon un ordre déductif nouveau et il a construit ce faisant une « doctrine logique et harmonieuse », l'hydrostatique. Le second et non le moindre mérite que Rougier reconnaît à Pascal est d'avoir, toujours dans ses traités posthumes, rejeté le principe scolastique selon lequel « les éléments ne pèsent pas en eux-mêmes », l'air dans l'air, ou l'eau dans l'eau par exemple. Le Traité de l'équilibre des liqueurs est du coup affecté d'une portée historique considérable, puisque la découverte des lois de la statique des fluides a ruiné «les principes de la dynamique du Stagirite », 《a porté le coup suprême à la physique scolastique », et écrit ainsi un chapitre essentiel de l'histoire de la statique des fluides.

À la différence de Félix Mathieu, Rougier est donc disposé à reconnaître le génie scientifique de Pascal, pour la raison majeure que Rougier a entamé contre la scolastique un combat qui n'est pas celui de l'homme de lettres. Une autre raison contribue à distinguer la position de Rougier de celle de Félix Mathieu. Aux critiques que lui a adressées et au pro-

58. [Rougier 2010, 232]. Cette formule vise à l'évidence Émile Boutroux.

59. À ce sujet, voir [Rougier 2010, 207]. Les erreurs contenues dans cette lettre, où Pascal se disculpait de l'accusation d'avoir pillé Torricelli sans le dire, sont unanimement reconnues, par Brunschvicg notamment qui tenait cette lettre, se réjouit Rougier, pour un «tissu d'inexactitudes », [Rougier 2010, 217]. 
cès que lui a intenté Léon Brunschvicg, Félix Mathieu a choisi de ne pas répondre ${ }^{60}$. Rougier, tout au contraire, s'en prend aux défenseurs modernes de Pascal. Parmi ces défenseurs, il cite Havet, Brunschvicg, Duhem, le P. Thirion, Milhaud, Strowski ${ }^{61}$, ce « Saint-Office universitaire » qu'il dénoncera à nouveau, nous l'avons vu, dans l'article du Mercure de France.

Il polémique surtout ouvertement contre Brunschvicg, auquel il consacre le chapitre IX : "L'exégèse et la dialectique de M. Brunschvicg ». Léon Brunschvicg a en effet répondu aux accusations de Félix Mathieu à trois reprises au moins : dans la Correspondance de l'Union pour la vérité, dans Le journal des Débats et dans la préface du tome I des Euvres de Pascal. Or Rougier a rencontré l'homme de lettres, qui lui a indiqué ce qu'il nomme euphémiquement « les erreurs de lecture » de Brunschvicg. Pour Rougier, ces 《erreurs de lecture »sont incontestables. Il réfute donc à son tour Brunschvicg, lui reproche ses «inconséquences », ses 《lectures hasardeuses », pire ses «falsifications », ses «altérations », ses modifications des textes. Notamment la suppression du primo contenu dans le texte de Jean Pecquet, qui attestait la priorité d'Auzoult dans la réalisation de l'expérience du vide dans le vide ${ }^{62}$. Rougier a repris cette accusation dans l'article du Mercure de France. Cependant, dans De Torricelli à Pascal, il conserve une certaine prudence dans l'énoncé de ces accusations, par comparaison évidemment avec les attaques ad hominem qu'il n'hésitera pas à lancer ultérieurement. S'il moque «l'exégèse et la dialectique de l'éminent éditeur de Pascal », que remplacera la très regrettable 《dialectique talmudique », ou encore les 《hardiesses » de son « exégèse pieuse », s'il l'accuse de déployer «toutes les ressources de la casuistique pascalienne », il reste au seuil des critiques beaucoup plus violentes qu'il lui adressera ultérieurement.

Ainsi, Pascal constitue bien l'une des cibles de la critique de Rougier, mais celui-ci s'en prend également aux « défenseurs modernes de Pascal ». Preuve que ce qui lui importe finalement autant que de retracer l'histoire de l'une des plus importantes défaites de la scolastique, c'est

60. Il a répondu à certaines critiques dans la série d'articles de 1907, puis a renoncé à polémiquer.

61. En ce qui concerne Duhem, il est excessif de le ranger parmi les défenseurs de Pascal puisque nous l'avons vu, il s'attache surtout à convaincre de l'importance du rôle joué par Mersenne. Voir également ce que nous avons écrit à ce sujet dans la n. 28.

62. À propos de l'expérience du vide dans le vide, Pecquet écrivait en effet : «tentatum primo feliciter acutissimi Auzotii sagacitate, experimentum condocefacere » [Pecquet 1651]. Jean Mesnard reproduit le texte de Pecquet [Mesnard 1970, 767]. Ce passage a donné lieu à différentes traductions et interprétations, destinées à minimiser sa portée. Rougier en parle très précisément. 
l'occasion qui lui est offerte de ferrailler contre Pascal ou plus exactement contre les pascaliens, Brunschvicg en tête. Et l'on doit maintenant se demander pourquoi ce combat contre Pascal et les pascaliens.

\section{2 - « L'affaire Pascal » dans son contexte}

Sans doute ne peut-on cependant éclairer parfaitement les positions ou la position prise par Rougier dans «l'affaire Pascal» que si l'on examine maintenant cette dernière de plus près en la situant dans son contexte.

Nous l'avons déjà souligné, la série d'articles publiés par Félix Mathieu, [Mathieu 1906], a opéré une rupture assez nette avec une tradition historiographique antérieure. La question de la priorité respective de Pascal et de Descartes avait certes déjà été posée, en raison des affirmations de Baillet et du père Daniel, mais jamais sur un ton aussi violemment polémique. Surtout, jamais personne n'avait suspecté l'authenticité de la lettre de Pascal de septembre 1647. On ajoutera que si l'accusation de faux conclut et couronne en quelque sorte les articles de Félix Mathieu, cette accusation n'est que l'accusation principale dont Pascal fait l'objet. Tout l'article n'est en fait, nous l'avons déjà souligné, qu'un long et minutieux réquisitoire dressé à son encontre. Il est donc temps de se demander pourquoi ce réquisitoire, et pour répondre à cette question, il faut s'interroger sur son contenu et sur son auteur, le premier permettant de se faire quelque idée du second, et d'aider à cerner son identité tout en dessinant rapidement le contexte historique dans lequel « l'affaire » a éclaté.

On ne sait pas grand chose en fait de ce Félix Mathieu, que ses contemporains paraissent fort bien connaître, et du coup ne se croient pas obligés de préciser son identité, sinon de façon très allusive ${ }^{63}$. Ainsi, et ce sont les indications les plus détaillées que l'on puisse recueillir à son sujet, l'historien Gabriel Monod le désigne d'abord plutôt vaguement comme « un esprit chercheur, subtil et compliqué », puis plus exactement comme « un libre penseur ardent fort ennemi du mysticisme », [Monod 1907, 113] et il lui reproche sa « joie bizarre à trouver un faussaire dans un homme que nous sommes habitués à vénérer comme le plus illustre des mystiques et des apologistes du christianisme ${ }^{64} \gg$. À l'évidence, une

63. Je remercie vivement Sylvain Matton, Christiane Demeulenaere et Géraldine Caps de m'avoir aidée à glaner les quelques informations ici rassemblées.

64. [Monod 1907, 114]. Cette protestation, jointe à celle par exemple d'Abel Lefranc, qui s'indignait de la mise en accusation de Pascal avec des accents très moralisateurs, aide à comprendre la réaction de Rougier face à ce qui lui apparaissait comme un «Saint-Office universitaire », même si, nous l'avons déjà dit, les universitaires n'ont pas unanimement rejeté les thèses de Félix Mathieu. 
notice nécrologique du journal Le Temps, parue en 1935 [Anonyme 1935], concerne l'auteur des articles:

On annonce la mort, à l'âge de 74 ans, de Félix Mathieu, savant et penseur original, qui toute sa vie éprouva la volupté de la recherche. Toute autre préoccupation que de poursuivre l'étude des idées était devenue pour lui secondaire. Chaque jour, depuis bien des années, dès l'ouverture de la Bibliothèque nationale, il s'asseyait à la même place que les habitués lui réservaient et où longtemps ils le chercheront. Jusqu'à cinq heures, on le voyait penché sur de vieux livres si totalement enfoncé dans sa lecture que des amis qui lui frappaient sur l'épaule ne parvenaient qu'à grand peine à l'en arracher. Sous ce large front s'évoquaient des milieux, des personnages de toutes sortes, Montaigne, Descartes, Mersenne, Huygens, Tartuffe - le vrai Tartuffe qu'il croyait avoir démasqué, Jean-Jacques Rousseau, J. de Maistre. C'est tout un monde, ressuscité par lui, que la flamme de l'incinération a dévoré avec ce cerveau.

L'indication selon laquelle il a été incinéré, acte quasiment militant à son époque, tend à confirmer sa qualité de « libre penseur » résolu. Une qualité qui trouve une autre confirmation dans le premier article de 1906, où Félix Mathieu dénonce, à propos de l'affaire saint Ange, un capucin, précise-t-il, que Pascal «a entrepris de faire brûler », le « zèle inquisitorial » de Pascal et de son père, qui lui a prêté main forte, et l'explication de ce zèle est simple : « derrière le semi-rationalisme de saint Ange, ils voient poindre le déisme du XVIII ${ }^{\mathrm{e}}$ siècle $^{65} \gg$. Dans un Livre d'hommage des lettres françaises à Émile Zola, on trouve dans les listes des protestataires « contre la violation des formes juridiques au procès de 1894 et contre les mystères qui ont entouré l'affaire Esterhazy », et qui «persistent à demander la révision ${ }^{66} »$, «Félix Mathieu, licencié ès lettres, ancien président de l'Association des étudiants de Paris ». On peut donc conclure que Félix Mathieu était un homme de lettres, un érudit ${ }^{67}$, mais

65. L'affaire saint Ange s'est déroulée à Rouen. Avec deux amis, Hallé de Monflaines et Adrien Auzoult, Pascal avait accusé un prêtre, Jacques Forton, en religion frère saint Ange, de professer un rationalisme théologique, qui le conduisait, par exemple, à tenter de démontrer la Trinité par raisons naturelles. Déjà sans doute converti au jansénisme, Pascal refusait ce rationalisme d'inspiration peut-être thomiste.

66. [Alexis, Séverine \& Labori 1898].

67. Cette qualité est corroborée par le témoignage de Lucien Febvre qui, évoquant le souvenir d'Henri Berr, parle incidemment de Félix Mathieu : 
aussi un rationaliste, un libre penseur et un dreyfusard, par conséquent aussi sans doute un républicain de tendance radicale.

Son intervention tonitruante dans le débat autour de l'expérience du puy de Dôme en 1906 n'est donc pas l'effet du hasard, surtout si l'on prend maintenant en considération le contexte historique de « l'affaire », [Mollier \& Georges 1994]. Ce contexte est en effet par excellence un contexte de tension, engendré par la multiplication des clivages au sein de la société de l'époque, des clivages en outre particulièrement complexes, ce qui explique en retour la complexité des différentes prises de position dans l'affaire Pascal. Sur le plan politique, l'affaire Dreyfus a opposé dreyfusards et anti-dreyfusards. Accusé et condamné en 1894 pour trahison, Dreyfus a été gracié en 1899 et réhabilité en 1906. Cette proximité de l'affaire explique sans aucun doute le fait que Brunschvicg puis Gabriel Monod n'aient pas hésité à instaurer une comparaison avec l'affaire Pascal, dŠautant que cŠest une accusation de faux qui est au centre de chacune dŠelle. Brunschvicg, nous l'avons vu, a le premier procédé à cette comparaison et invité à identifier Pascal et Dreyfus, tous les deux injustement condamnés ${ }^{68}$. Et selon Gabriel Monod, qui s'inspire des conclusions de l'examen critique des textes entrepris par Abel Lefranc,

M. Mathieu a été victime d'une hallucination analogue à celle des accusateurs de Dreyfus qui, prenant pour point de départ la certitude qu'il avait écrit le bordereau, ont vu tout se transformer à leurs yeux en preuves de la justesse de cette attribution. [Monod 1907, 115]

Félix Mathieu est ainsi accusé de faire preuve d'un aveuglement comparable à celui des anti-dreyfusards et de donner un exemple « de la passion qui depuis quelques temps s'introduit dans la discussion des questions scientifiques »: occasion pour Monod de tirer une leçon de méthode

En face de lui, souvent, Félix Mathieu, «le Mathieu de Pascal», un de ces puits de science insondables, mais qui ne rendent jamais leur eau. [Febvre 1953, 340]

Ce témoignage est à son tour corroboré par celui d'Ernest Tonnelat qui met dans l'entourage de Charles Andler «F. Lot, Georges Pariset, Seignobos, Camille Bloch; le sociologue Marcel Mauss; le philosophe André Lalande; Félix Mathieu, qui dépensait dans sa conversation étincelante les trésors qu'il n'a jamais su enfermer dans des livres », [Tonnelat 1937, 128]. Ces deux témoignages aident à comprendre pourquoi Félix Mathieu ne doit sa renommée qu'à ses seuls articles de la Revue de Paris. Merci à Sylvain Matton pour m'avoir signalé ces deux passages.

68. Même si ce n'est pas exactement ce que dit Brunschvicg, il est difficile de ne pas interpréter ainsi la longue allusion qu'il fait à l'affaire. $C f$. plus haut, n. 31 . 
historique et de «mettre en garde les jeunes historiens contre l'introduction de passions étrangères à la science dans l'examen de questions scientifiques », [Monod 1907, 115].

Quand Rougier évoque à son tour l'affaire Pascal, il montre qu'il a très bien perçu cette dimension du débat, qui n'oppose cependant en la circonstance que des dreyfusards. Mais ce débat est en outre déclenché en plein cœur des affrontements entre républicains laïques et catholiques conservateurs, affrontements qui se sont conclus en 1905 par la séparation de l'Église et de l'État. Enfin, sur le plan philosophique, en réaction contre les progrès du naturalisme, du matérialisme et du scientisme, jugés dangereusement menaçants, deux grands courants dominent tous les autres et s'affrontent : d'une part le spiritualisme, qui est en train de devenir peu ou prou la philosophie officielle de l'Université, et qu'incarnent ou que défendent les quatre grands « $\mathrm{B} »:$ Brunschvicg, Bergson, Blondel, Boutroux ; et d'autre part, le thomisme, c'est-à-dire la philosophie officielle de l'Église catholique. Le spiritualisme universitaire n'a rien d'univoque et l'on peut repérer en son sein des tendances, des courants ; pour simplifier, une aile droite et une aile gauche. On peut distinguer d'un côté des spiritualistes comme Brunschvicg, qui représente ou incarne un courant rationaliste, laïque et démocratique, un courant qui est parfois proche du républicanisme radical. Ce courant fait place à une réflexion sur les sciences et donne lieu, au plan de la philosophie et de l'histoire des sciences, à des travaux importants, [Sève 1962]. D'un autre côté, des philosophes comme Boutroux ou Bergson représentent un courant plus mystique, plus confessionnel, plus conservateur. Contraints cependant de prendre acte de l'essor des sciences, ils s'efforcent, comme le fait Boutroux, de les adapter à la métaphysique et à la religion en soulignant les limites de la connaissance scientifique. Mais ce spiritualisme peut prendre aussi la forme plus offensive d'un anti-positivisme, d'un anti-intellectualisme qui se complaît à développer le culte de l'intuition, et à prôner la méthode de sympathie, comme le fait Bergson. Entre ce spiritualisme et l'Église catholique, l'accord est pourtant loin d'aller de soi. D'une part, parce que même s'il reconnaît l'existence de Dieu et l'immortalité de l'âme - c'est ce qui le définit comme spiritualisme - , il peut aussi déboucher aisément sur le déisme. D'autre part, parce que si le thomisme est bien la philosophie officielle de l'Église catholique, celle-ci ne peut que prendre ses distances à l'égard d'un anti-rationalisme ou d'un anti-intellectualisme jugés étrangers au thomisme et contraire à la tradition ultramontaine. Pour autant, ce spiritualisme est loin de rebuter tous les catholiques, au total très divisés ${ }^{69}$. Ces clivages se re-

69. Sur ce contexte idéologique, voir l'ouvrage très éclairant de Lucien Sève [Sève 1962]. Voir également Jean-Louis Fabiani [Fabiani 1988]. 
trouvent par ailleurs sur un plan plus littéraire, et revêtent une forme spécifique, qui nous ramène directement à l'affaire Pascal. Ceux qu'Antoine Compagnon nomme en effet les « antimodernes », Georges Sorel, Péguy, Édouard Berth, Henri Massis, Barrès, investissent en effet alors la figure de Pascal, au détriment de celle de Descartes ${ }^{70}$. Emblème du refus de l'intellectualisme, tel que le théorise Bergson, Pascal incarne pour tous ces auteurs l'anti-Descartes. Pascal dont, au prix d'une interprétation très simplificatrice de sa pensée, ils retiennent essentiellement l'humiliation de la raison et l'exaltation du sentiment, et qu'il devient dès lors possible de transformer en porte-parole du combat contre le rationalisme.

On comprend par là même aisément que l'intervention de Félix Mathieu ait pu passer à l'époque, et Rougier se fait l'écho de ces rumeurs, pour le résultat d'un complot, «ourdi dans les couloirs de l'École ${ }^{71}$ », un complot visant Pascal, via Émile Boutroux, l'un des piliers du spiritualisme universitaire, non seulement co-éditeur avec Brunschvicg, ainsi que nous l'avons vu, des (Euvres de Pascal, mais aussi auteur de ce petit ouvrage consacré à Pascal contre lequel Rougier polémiquait. Un Émile Boutroux qui dispensait à l'École un cours sur Pascal, un cours qui a sans doute inspiré son ouvrage, et suscité une vive protestation de la part de certains de ses collègues. Un Émile Boutroux transformé dès lors par ces derniers en un «épouvantail ». D'où le fameux « complot» dont Félix Mathieu aurait été l'âme ou plus exactement la plume. C'est Péguy qui délivre ces informations, lorsqu'il évoque ses souvenirs de normalien ${ }^{72}$ au moment où après avoir été dreyfusard, militant socialiste et anticlérical, il est retourné au catholicisme. Selon lui, un des maîtres de l'École ${ }^{73}$,

70. [Compagnon 2007]. On y découvre l'éventail, très complexe, des différentes positions prises à l'égard de Pascal entre 1900 et 1930.

71. «S'il faut en croire M. Massis, il aurait été l'âme d'un noir complot, ourdi dans les couloirs de l'École normale, qui tourna à son entière confusion », [Rougier 2010, 49].

72. Charles Péguy avait intégré l'École normale en 1894.

73. Il n'est pas impossible qu'il s'agisse de Charles Andler, maître de conférences, puis professeur adjoint à l'École normale, un ami de Lucien Herr, le bibliothécaire de l'École, qui avait converti Péguy au socialisme et au dreyfusisme, avant que l'évolution intellectuelle et politique de Péguy ne l'éloigne de son maître; ou plus probablement de Lucien Herr lui-même, selon l'hypothèse que m'a suggérée Sylvain Matton et que pourrait corroborer un passage de Léon Blum :

Herr était chez moi à la fin de l'après-midi, quand les nouvelles nous furent apportées toutes chaudes par un de ses amis, nommé Félix Mathieu, garçon bizarre et plein de verve, qui avait pris Pascal en haine particulière, et qui consacra plusieurs articles de la Revue de Paris, à établir ses plagiats scientifiques. [Blum 1965, 566] 
qu'il ne nomme pas, aurait affirmé, à propos de Pascal : « cette fois-ci, nous le tenons, nous aurons sa peau ». Et il poursuit :

C'était au seuil de cette petite conjuration, de cette petite cabale qui fut tramée à l'École normale et dans le milieu de l'École normale par un nommé Mathieu ou Matthieu pour démontrer que Pascal était le dernier des faussaires et qui n'a pas laissé de traces. Ni la cabale ni Mathieu.

La suite ne manque pas non plus d'intérêt :

M. Lavisse naturellement avait prêté la Revue de Paris ${ }^{74}$ pour la perpétration de cet attentat. Quand il vit que cela tourna mal, lâche dans le crime même, il lâcha ses complices et déclara qu'il ne s'était pas aperçu qu'on faisait ça. [Péguy $1965,994^{75}$ ]

Péguy avait déjà évoqué ce qui est pour lui «l'affaire Mathieu» en 1907, dans Un poète l'a dit, où il montrait comment, toujours dans le milieu de l'École normale, Boutroux était perçu comme un conservateur, un adversaire, un ennemi, « chargé de conserver toute la société bourgeoise » :

Nous avons vu régler sans lui cette affaire Mat[t]hieu. Et pourtant, il avait vécu plusieurs années de Pascal, [...]; il en avait fait toute une série de cours en Sorbonne auxquels nous allions religieusement assister. Il en fait un livre, je crois depuis chez Hachette. S'il a fait tout ce travail, tous ces différents travaux, s'il a tant approfondi la question sans y rencontrer le puy de Dôme, comment travaillait-il? Et s'il a trouvé, dès lors et depuis, qu'il n'y a pas le puy de Dôme, comment s'est-il tu et se tait-il? [Péguy 1965, 923]

Le ton est plus violent dans De la situation faite à l'histoire et à la sociologie, où il évoque :

... le scandale, d'ailleurs parfaitement inutile, provoqué par cet imposteur de Mathieu ou Matthieu, un des plus grands

\footnotetext{
On remarquera que Léon Blum paraît tenir pour acquis qu'il y a bien eu plagiat de la part de Pascal! On soulignera d'autre part que Lucien Herr était le secrétaire de la Revue de Paris.

74. L'historien Ernest Lavisse dirigeait la Revue de Paris depuis 1904.

75. Un nouveau théologien, M. Fernand Laudet, le texte est de 1911.
} 
imposteurs que la terre ait jamais porté. Je parle naturellement de Mathieu qui n'est ni évêque ou archevêque, au moins dans l'Église romaine apostolique, ni cardinal, ni membre de l'Académie française. [Péguy 1965, 1000]

Pour Péguy, les articles de Félix Mathieu relevaient donc d'une conjuration et avaient pour but d' ' avoir la peau » de Pascal, et cela pour des raisons essentiellement politiques. En ce qui concerne le but poursuivi, on peut assez aisément le croire, au vu du ton de ces articles. La thèse du « complot» reste quant à elle hypothétique. Les commentaires de Péguy permettent surtout d'interpréter ces articles comme une réaction des républicains laïques et rationalistes à la tendance mystique confessionnelle et conservatrice du spiritualisme universitaire, dont le ralliement autour de la figure de Pascal constituait, nous l'avons dit, un marqueur tout à fait significatif : ce n'est donc pas un hasard si le choix de Félix Mathieu s'est porté sur Pascal. Ce n'est pas non plus un hasard si Rougier, hostile à Bergson, à l'intuition et à la méthode de sympathie comme méthode de connaissance ${ }^{76}$, sans épouser intégralement toutes ses thèses, et tout en se situant sans doute sur le plan politique, sur des positions très éloignées de celles de Félix Mathieu ${ }^{77}$, se sent plus proche de ce dernier, avec lequel il partage au moins un même athéisme, un même anticléricalisme, que de ses adversaires pascalisants ou pascaliens. Tous, plus ou moins spiritualistes, anti-intellectualistes, anti-positivistes et cléricaux ${ }^{78}$. On peut deviner également les motifs plus spécifiquement épistémologiques de l'hostilité manifestée par Rougier à leur égard : via l'influence de Taine et de Renan, Rougier se rattache à la tradition positiviste, ce qui l'a conduit à manifester très tôt, et l'un des premiers en France, son intérêt pour le positivisme logique. En revanche, la focalisation de sa

76. De Torricelli à Pascal égratigne également au passage cette méthode de sympathie, par exemple [Rougier 2010, 92].

77. Sur les positions politiques de Rougier, sur son appartenance à la droite, voire à l'extrême droite, qui l'a au minimum considéré comme l'un de ses inspirateurs, sur sa défense du libéralisme économique, sur ses positions à l'égard du gouvernement de Vichy durant la seconde guerre mondiale, qui ne sauraient ici être prises en considération puisqu'elles sont largement postérieures à la période qui nous intéresse, voir [Pont \& Padovani 2007].

78. Dans ce contexte, on ne s'étonnera pas que Abel Rey, très laïque et anticlérical soit à peu près le seul à avoir pris le parti de Félix Mathieu. L'article d'Abel Lefranc, de son côté, atteste exemplairement ce que sans doute Rougier considérait comme le cléricalisme de certains partisans de Pascal. Ces différentes prises de position illustrent jusqu'à la caricature le partage idéologique qui a pu se produire dans «l'affaire Pascal ». Il faudrait évidemment, répétons-le, une étude plus fine des positions des uns et des autres, qui ne relèvent pas toutes de cette présentation un peu sommaire. On trouve d'autre part une illustration de cette critique de l'anti-intellectualisme dans l'article de Rougier [Rougier 1931a]. 
critique sur Brunschvicg s'explique plus malaisément. Sur le plan épistémologique, Rougier n'est pas entièrement éloigné de celui auquel il a sans doute emprunté la thèse de la mobilité et de la plasticité de la raison. Mais en même temps, il est vrai, son empirisme et son attachement au formalisme logique l'en éloignent. Autant, sur le plan politique, que son anti-républicanisme, qui a permis à la «nouvelle droite »d'Alain de Benoist de le revendiquer bien des années plus tard comme l'un de ses inspirateurs. Cet éloignement s'est en outre très probablement renforcé des raisons plus personnelles que nous avons déjà évoquées.

\section{5 - Conclusion}

On le voit, l'intérêt du manuscrit aujourd'hui publié dépasse largement une histoire des sciences entendue au sens étroit du terme. Il faut peutêtre cependant dire en conclusion quelques mots de la façon dont Rougier a pratiqué une discipline qui ne lui était nullement familière. Il a réellement pris la peine d'effectuer une recherche très précise sur les faits et les théories dont il relate l'histoire, et son récit constitue bien à part entière un document qui prend place dans la longue histoire de la façon dont, en Italie d'abord puis en France, a été traitée au milieu du XVII ${ }^{\mathrm{e}}$ siècle les questions du vide et de la «pesanteur et pression de l'air ». Il n'apporte il est vrai sur cette question aucun élément nouveau, et son interprétation de cet épisode fameux de l'histoire des sciences est pour une part dépendante de celle de Duhem : elle demeure cependant largement originale et mérite amplement d'être découverte. Le lecteur risque cependant d'être surpris par sa tendance regrettable à formuler de temps à autres les positions scientifiques qu'il expose en termes anachroniques, à commettre en d'autres termes le péché capital de récurrence : ainsi n'hésite-t-il pas, par exemple, à invoquer la théorie de l'élasticité des gaz pour désigner la théorie professée par Roberval sur la «raréfaction de l'air », ou à souligner que Pascal ignorait, c'est une évidence, la loi de Mariotte, ou à invoquer la «théorie des phases de Gibbs » pour rendre compte de certains résultats d'expérience. Ce défaut ne grève pas cependant lourdement la qualité du texte.

Toutefois, on ne peut nier que le principal intérêt de celui-ci provient de la façon dont Rougier a mêlé des considérations très actuelles à un récit destiné prioritairement à illustrer la déroute de la scolastique dans le champ de la philosophie de la nature. Non seulement De Torricelli à Pascal constitue de ce point de vue un document très caractéristique de la personnalité intellectuelle de Rougier, mais c'est sans doute sur 
la querelle du début du $\mathrm{xx}^{\mathrm{e}}$ siècle que De Torricelli à Pascal apporte l'éclairage le plus intéressant. Cette méta-querelle n'a jamais été étudiée de façon systématique ${ }^{79}$, pour des raisons que l'on peut deviner. Il est impossible en effet, et la lecture de De Torricelli à Pascal l'atteste, de l'exposer sans entrer dans le détail de considérations factuelles extrêmement précises et détaillées, d'aucuns diront fastidieuses : quand Florin Périer a-t-il rencontré Pascal à Paris vers la fin de l'année 1647? À quelle date le même Florin Périer s'est il trouvé en mesure de réaliser à Clermont l'expérience que son beau-frère lui a demandé d'effectuer dès septembre 1647, aux dires de Pascal? Quel laps de temps désigne la formule «ces jours passés »: il y a une semaine, quelques semaines, plusieurs semaines? Sur chacune de ces questions, et sur bien d'autres, tous ceux qui ont pris position, avant et surtout après l'intervention de Félix Mathieu, ont proposé leur propre interprétation, qui requiert chaque fois une exégèse minutieuse des documents permettant de construire cette interprétation. Si l'on peut hésiter à entreprendre la comparaison systématique de ces variations interprétatives, l'intérêt serait grand pourtant d'éclairer ces différentes lectures en prenant en compte l'identité, au sens large du terme, de chacun des protagonistes, comme nous avons tenté de le faire pour une partie d'entre eux, c'est-à-dire en essayant de déterminer la façon dont chacun se positionnait en son temps dans le champ philosophique et idéologique. À l'étude des controverses, nul doute qu'une telle recherche apporterait des éléments précieux. En tout état de cause, l'intérêt de l'intervention de Rougier consiste dans l'éclairage singulier qu'elle apporte sur un moment de l'histoire de la philosophie française sur lequel, et c'est son second grand mérite, elle nous invite à nous repencher.

Rougier n'a pas renouvelé cette incursion dans un champ disciplinaire qui n'était quand même pas le sien, ce qui confirme à la fois qu'il était plus philosophe qu'historien des sciences, et que cet ouvrage a bien été un ouvrage de circonstance. Cela n'enlève rien à l'intérêt d'un texte qui, par son ton, par son engagement polémique, autant que par ses orientations philosophiques, fait intégralement partie d'une œuvre dont l'éclectisme ne doit pas conduire à sous-estimer la possible cohérence, qu'il atteste à sa façon en dépit de sa singularité.

79. J'y ai fait moi-même à plusieurs reprises allusion, voire traité certains de ses aspects sans l'étudier jamais en tant que telle. 


\section{Note sur l'édition}

Le manuscrit de Louis Rougier, tel qu'il nous est parvenu, présente un bon nombre d'imperfections qui empêchaient de l'éditer en l'état, sans remettre pour autant en question l'opportunité de cette édition.

D'abord, six pages manquent, ce qui ne nous a pas paru un argument suffisant pour renoncer à la publication, dans la mesure où l'absence de ces pages ne grève pas la compréhension du texte dans son ensemble. Plus grave apparemment, manquent également deux parties du chapitre XI, qui figurent dans la table des matières proposée par Rougier. En ce cas encore cependant, l'absence de ces développements, qui ne représentaient sans doute qu'un petit nombre de pages, ne nous a pas paru susceptible de compromettre la compréhension globale du texte.

Quelques mots n'ont pas été reproduits, et apparaissent sous la forme d'un blanc dans le manuscrit. Nous avons choisi de proposer entre crochets les mots qui ont été omis, en les faisant suivre d'un point d'interrogation.

À la page 223 du dactylogramme, Rougier a laissé également un blanc dans le manuscrit à la place du principe de statique énoncé par Descartes qu'il se proposait de citer : nous avons en ce cas rempli à sa place le blanc qu'il avait omis de combler.

Le texte d'une note s'est révélé illisible. Rougier a aussi parfois oublié de renseigner quelques-uns de ses appels de note : nous signalons l'absence de la note annoncée, que nous avons cependant renseignée chaque fois que cela a été possible. D'une façon générale, la façon dont Rougier a rédigé ses notes est assez peu rigoureuse : nous avons donc procédé à un certain nombre de corrections, sans pour autant corriger en toute circonstance cette absence de rigueur, dans la mesure où les notes telles qu'elles sont composées restent fonctionnelles.

On relève dans l'exemplaire dactylographié original un certain nombre de fautes de frappe, qui conduisent par exemple à inverser l'ordre des lettres dans un mot. Nous avons corrigé ces coquilles sans les signaler. On relève aussi des fautes d'orthographe, qui n'ont cependant pas toutes le même statut, c'est pourquoi nous les avons traitées différemment. Quelques-unes sont des fautes d'accent ou encore des fautes que l'on peut interpréter comme des fautes de lecture de la part de l'auteur de la dactylographie : ainsi, Pierius est constamment orthographié Pierins, le $\langle\mathrm{u} »$ ayant sans doute était confondu avec un $\langle\mathrm{n} » ;$ Jaloustre est orthographié Saloustre, en raison sans doute également de la ressemblance entre le $\langle s »$ et le $\langle\mathrm{j} \gg$. Il est vrai toutefois que le même nom est orthographié par erreur Laloustre dans l'article de Rougier paru dans le 
Mercure de France dont il est longuement question dans notre introduction. Nous avons choisi de corriger les fautes d'accent et de rétablir la bonne orthographe des noms propres sans le signaler.

En ce qui concerne encore l'orthographe des noms propres, le manuscrit dactylographié révèle parfois des hésitations : ainsi Auzoult est orthographié Auzoult ou Auzout, Baliani est orthographié soit Baliani, soit Baliano, Otto de Guericke est une fois orthographié Otto Guericki : nous avons harmonisé ces différentes orthographes et nous les avons corrigées chaque fois que nécessaire sans juger non plus indispensable de le signaler.

On peut aussi relever un désaccord entre deux orthographes d'un même mot : ainsi le puy de Dôme est orthographié par Rougier, mais aussi par la plupart des auteurs cités, Puy-de-Dôme. Nous avons adopté dans notre introduction l'orthographe actuelle et respecté l'orthographe antérieure dans les textes cités, soit par Rougier, soit par nous-même dans notre introduction. Nous avons de même conservé l'orthographe Des Noyers, qu'on écrit aujourd'hui plutôt Desnoyers.

Enfin, nous avons régularisé l'emploi des majuscules.

Dans l'Avertissement ainsi que dans la Conclusion, Rougier a porté quelques corrections manuscrites sur l'exemplaire dactylographié. Même si ces corrections ne présentent pas un intérêt décisif, nous avons choisi de les indiquer. Dans certains cas, Rougier a modifié le texte dactylographié : dans ce cas, nous avons choisi de retenir le texte corrigé, et d'indiquer dans une note et entre crochets la version initiale. Parfois, quelques mots ont été purement et simplement supprimés : en ce cas, nous indiquons également dans une note et entre crochets les mots supprimés. Enfin, les termes entre crochets non suivis d'un point d'interrogation ont été rajoutés par Rougier.

Le manuscrit dactylographié ne comporte pas de bibliographie, mais seulement une très succincte liste des abréviations utilisées. Nous proposons donc une bibliographie d'ensemble, qui vaut aussi bien pour notre introduction que pour le texte de Rougier lui-même. En ce qui concerne l'œuvre de Rougier, nous n'avons pas jugé utile d'en proposer une bibliographie complète : le lecteur pourra consulter celle-ci dans les deux volumes de Philosophia Scientice qui ont déjà été consacrés à la vie et à l'œuvre de Rougier [Pont \& Padovani 2006, 2007].

Enfin, quatre planches dont le contenu est indiqué par Louis Rougier devaient accompagner le texte. Une seule de ces planches a été retrouvée dans le manuscrit, celle où est représentée, dit la légende, «L'expérience du vide dans le vide selon le Traité de la pesanteur de la masse de l'air de 
Blaise Pascal ». En réalité, la figure contenue dans ce Traité est beaucoup plus sommaire, et nous ne connaissons pas l'origine de la gravure reproduite par Rougier. Nous avons donc ajouté la représentation de cette expérience telle qu'on la trouve dans le Traité de Pascal (Planche 1bis) à celle retrouvée dans le manuscrit. Pour les trois autres planches, nous ne possédions que leur légende. La première se trouve dans la Technica curiosa de Gaspard Scott, et nous la reproduisons grâce à l'aimable autorisation de Monsieur le conservateur de la Bibliothèque municipale de Nancy et grâce à Jean-Paul Rothiot qui a bien voulu se charger de la numériser. La quatrième est due à Pierre Duhem, qui a reconstitué l'expérience, et on la trouve dans l'ouvrage de Fortunat Strowski, Pascal et son temps. Enfin, la légende de la planche 3 indiquait « Expérience du vide dans le vide attribuée à Pascal par le père Noël dans la Gravitas comparata et reconstituée par Pierre Duhem ». Il semble qu'il y ait eu ici une confusion de la part de Rougier, car c'est le père Thirion qui a reconstitué cette expérience dans l'un de ses articles consacré à Pascal. C'est cette reconstitution par conséquent que nous avons choisi de reproduire.

Enfin, nous avons ajouté le schéma dont Descartes avait illustré la lettre citée page 74 et qui ne figure pas dans le manuscrit de Rougier, dans la mesure où ce schéma nous a paru apporter un éclairage indispensable à la compréhension du texte de Descartes.

Ma gratitude va à Sandrine Avril pour la composition du texte de cette édition. Merci également à Jules Henri Greber pour sa relecture des épreuves du texte de Rougier ainsi qu'à Sylvain Matton pour sa relecture de l'introduction. 